\title{
Reciprocal figures, graphical statics and inversive geometry of the Schwarzian BKP hierarchy
}

\author{
B.G. Konopelchenkol and W.K. Schief \\ School of Mathematics, The University of New South Wales, \\ Sydney, NSW 2052, Australia
}

\begin{abstract}
A remarkable connection between soliton theory and an important and beautiful branch of the theory of graphical statics developed by Maxwell and his contemporaries is revealed. Thus, it is demonstrated that reciprocal triangles which constitute the simplest pair of reciprocal figures representing both a framework and a self-stress encapsulate the integrable discrete BKP equation and its Schwarzian version. The inherent Möbius invariant nature of the Schwarzian BKP equation is then exploited to define reciprocity in an inversive geometric setting. Integrable pairs of lattices of non-trivial combinatorics consisting of reciprocal triangles and their natural generalizations are discussed. Particular reductions of these BKP lattices are related to the integrable discrete versions of Darboux's 2+1-dimensional sine-Gordon equation and the classical Tzitzéica equation of affine geometry. Furthermore, it is shown that octahedral figures and their hexahedral reciprocals as considered by Maxwell likewise give rise to discrete integrable systems and associated integrable lattices.
\end{abstract}

\section{Introduction}

The soliton with its novel interaction property represents one of the most intriguing of nonlinear phenomena in modern physics. Solitons occur in such diverse areas as hydrodynamics, plasma and solid state physics, as well as in general relativity [1, 2, 3. 3. They have important current technological applications in optical fibre communication systems and Josephson junction superconducting devices [4, 5]. Nonlinear equations which describe solitonic phenomena (integrable systems) are ubiquitous and of great mathematical interest. Thus, in particular, they are generically amenable to the Inverse Scattering Transform (IST) method [6, 7] and admit invariance under Bäcklund transformations [8, 9] with associated nonlinear superposition principles (permutability theorems) whereby analytic expressions descriptive of multi-soliton interaction may be constructed.

Here, it demonstrated that there exists a deep connection between discrete integrable systems and reciprocal figures of graphical statics (structural

\footnotetext{
${ }^{\dagger}$ Permanent address: Dipartimento di Fisica, Università di Lecce and Sezione INFN, 73100 Lecce, Italy
} 
geometry). The theory of reciprocal figures was developed by the physicist and geometer James Clerk Maxwell in the XIX ${ }^{\text {th }}$ century in connection with diagrams of forces representing stresses in frameworks [10]. Maxwell's contributions [11] culminated in the paper On reciprocal figures, frames and diagrams of forces 12] for which he received the Keith Prize of the Royal Society of Edinburgh. However, Maxwell points out that the construction of diagrams of forces in which each force is represented by one line had been discovered independently and earlier by the practical draughtsman W.P. Taylor and he also refers to William Rankine's Applied Mechanics [13]. Subsequently, in [14], Fleeming Jenkin gave a variety of practical applications of reciprocal figures to the calculation of strains on frameworks. He received the Keith Gold Medal for his paper On the application of graphic methods to the determination of the efficiency of machinery [15. His contribution Bridges to the Encyclopaedia Britannica [16] contains reciprocal figures associated with a variety of bridges including suspension bridges. References to the relevant literature of this period may be found in Luigi Cremona's two treatises Le Figure Reciproche Nella Statica Grafica [17].

It is interesting to note that Karl Culmann in his Graphische Statik [18] makes great use of diagrams of forces, some of which are reciprocal. Culmann's student Maurice Koechling, in turn, was one of two chief engineers in Gustave Eiffel's company and carried out in a graphical manner many calculations for the Eiffel Tower built in 1889. Remarkably, a century later, Maxwell's observation that there exists a close relationship between plane rectilinear reciprocal figures and perspective representations of closed polyhedra was rediscovered in the context of 'artificial intelligence', namely the recognition and 'realizability' of plane line drawings as three-dimensional polyhedral scenes [19, 20].

The connection with integrable systems is made by identifying the geometric compatibility which guarantees the existence of reciprocal figures with the algebraic compatibility of linear difference equations which give rise to nonlinear integrable difference equations. We begin with the simplest pair of reciprocal figures, namely reciprocal triangles. It is demonstrated that the dilation coefficients which encode the reciprocal relation between the two triangles satisfy the integrable discrete BKP equation. The discrete BKP (dBKP) equation is known to encapsulate the entire $B$-type Kadomtsev-Petviashvili hierarchy of integrable equations 21] and may also be regarded as a nonlinear superposition principle for eight solutions of this hierarchy generated by Bäcklund transformations [22]. The vertices of the reciprocal triangles and their 'interior points' are shown to obey a simple 8-point relation which is likewise integrable and may be regarded as a 'Schwarzian' version of the dBKP equation. Since this 8-point relation is formulated in terms of two cross-ratios, it is invariant under the group of inversive transformations, that is Möbius transformations and complex conjugation. This observation is used to formulate reciprocity in a purely inversive geometric manner. Thus, the Schwarzian BKP equation constitutes a natural object of inversive geometry.

Integrable lattices on the complex plane consisting of reciprocal triangles and their 'circle geometric' extensions are defined in terms of pairs of face-centred cubic (fcc) lattices and it is shown that there exist canonical geometric reductions 
which are associated with the discrete analogue of Darboux's 2+1-dimensional sine-Gordon equation set down in 23] and the discrete Tzitzéica equation which has been obtained earlier in both an algebraic and purely geometric manner [24-27]. We discuss another class of reciprocal figures which is obtained via a 'truncation' of Maxwell's example of an 'octahedral' figure 10]. Once again, it is shown that the pair of reciprocal figures so obtained may be considered 'integrable'. We conclude the paper with a discussion of Maxwell's complete octahedral figure and determine a canonical class of hexahedral reciprocals which may be defined in terms of multi-ratio relations. This class is then shown to be associated with integrable lattices of octahedral-hexahedral combinatorics.

In an earlier paper [28], we established a connection between the Schwarzian Kadomtsev-Petviashvili hierarchy and Menelaus' fundamental theorem of plane geometry. Therein, we first set down the discrete Schwarzian KP equation in the context of soliton theory and then interpreted it geometrically. Here, we adopt an inverse procedure. We discuss the geometry of reciprocal figures and then retrieve discrete integrable systems and their associated hierarchies. This is to underline the intimate relation between canonical hierarchies of soliton theory and plane configurations in inversive geometry.

\section{Reciprocal figures, frames and diagrams of forces}

Some 140 years ago, James Clerk Maxwell [10] discovered a fascinating connection between planar reciprocal figures, diagrams of forces and orthogonal projections of polyhedra. Subsequently, Jenkin [14, 15] applied diagrams of forces and reciprocal figures to the most important cases occurring in practice at the time. Other names associated with the theory of graphical statics and, in particular, reciprocal diagrams of forces are Bow, Culmann, Cremona, Rankine and W.P. Taylor [13, 17, 18, 29]. Maxwell's geometric definition of a frame is a system of straight lines connecting a number of points. These lines may be thought of as material pieces such as beams, rods or wires and the forces which act on each piece joining two points may be interpreted as two forces of the same magnitude but opposite direction acting between the two points.

The simplest frame consists of four points joined by six lines as displayed in Figure 1 1 (a). One may now inquire as to whether there exist forces acting on the points along the lines such that the frame is in equilibrium. Figure 1(a) depicts six forces and their counterparts which are parallel to the lines. In order to verify that the frame is in equilibrium, it must be possible to rearrange any three force vectors which act on a point in such a way that they form a closed polygon. Figure 1 (b) indicates that the four polygons of forces associated with the points 1,2,3 and 4 are indeed closed. Thus, the frame is in equilibrium and any other forces which guarantee equilibrium must be multiples of those drawn in Figure 1(a). Hence, the ratios and the directions of the forces are determined. 


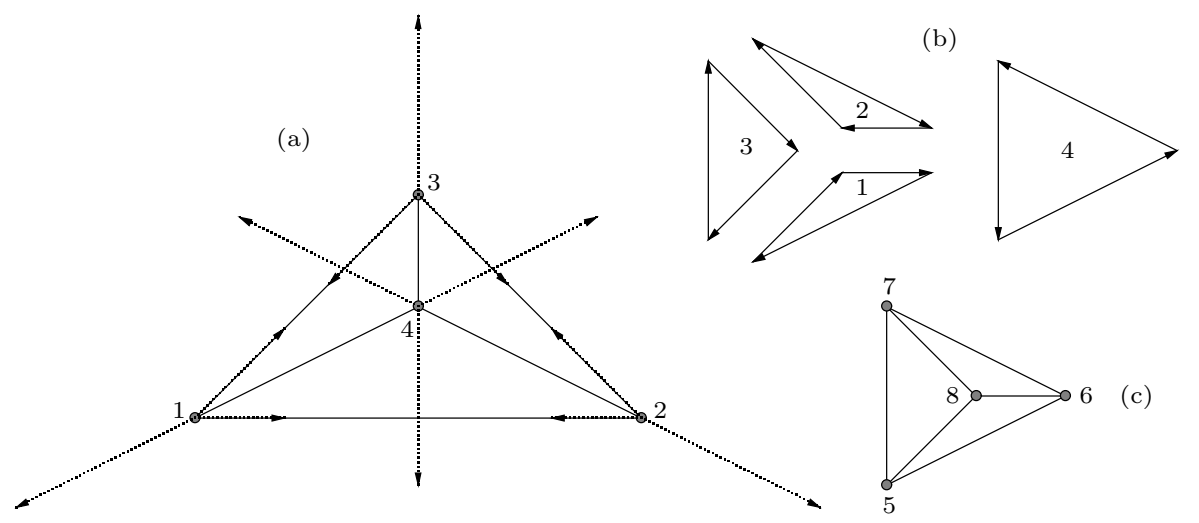

Figure 1: A frame and its reciprocal diagram of forces

Now, the crucial observation is that the four polygons of forces may be fitted together to form another frame. This is depicted in Figure 1 (c). Consequently, it is also possible to interpret the directed lines connecting the points $1,2,3$ and 4 as force vectors which act on the points of the second frame in such a way that equilibrium is maintained. Hence, the two figures 1 (a) and 1)(c) constitute reciprocal figures. Maxwell gave a beautiful geometric construction of the second frame (cf. Figure 2). Thus, if we draw four circles passing through any three points of the original figure then the line segments which connect the centres of these four circumcircles are orthogonal to the six lines and form another figure consisting of four points and six lines. If we rotate this figure by 90 degrees then a reciprocal figure is obtained. This figure is definite in size and position but any figure similar to it is still reciprocal to the original figure.

It has been seen that the reciprocal of a figure of the type 1(a) is uniquely determined up to a scaling and its position on the plane. Based on the following definition, Maxwell [10] investigated in detail the conditions of indeterminateness and impossibility in drawing reciprocal figures:

Definition 1 (Maxwell (1864)). Two plane figures are reciprocal when they consist of an equal number of lines, so that corresponding lines in the two figures are parallel, and corresponding lines which converge to a point in one figure form a closed polygon in the other.

He first analysed this problem in an algebraic manner. Thus, the construction of the reciprocal of Figure 1 (a) is possible and unique because the number of points equals the number of polygons in this figure. Figure 3 (a) displays a figure of 'octahedral' combinatorics. The number of polygons exceeds the number of points by two. Accordingly, the construction of a reciprocal figure is possible but there exist two degrees of freedom. This is reflected in the following construction of the reciprocal figure. We first draw three concurrent lines which are parallel to the edges 1,4 and 5 and arbitrarily choose a point on each line. The remainder of the reciprocal figure is then uniquely determined by drawing successively lines 


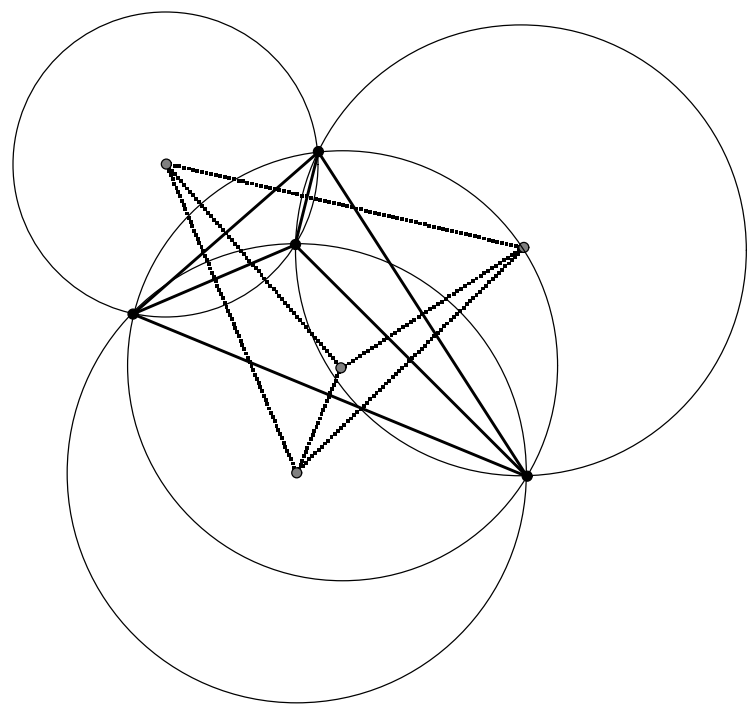

Figure 2: Geometric construction of reciprocal triangles

parallel to the edges $2,12,3,11,6,7,9$ and 8 . The latter two lines and the line which is parallel to the edge 10 and passes through the intersection of the lines parallel to the edges 6 and 7 may be shown to be concurrent. Hence, in addition to a scaling, the reciprocal figure is indeed capable of two degrees of variability. It is noted that the reciprocal figures 3 have the combinatorics of an octahedron and a cube respectively.

The construction of the reciprocal figure $3(\mathrm{~b})$ shows that if we begin with a figure of the combinatorics of Figure 3(b) then it is not always possible to construct a reciprocal. Indeed, there exist two conditions on the directions of the lines which are required to hold. From a physical point of view, this implies that if these conditions are satisfied then the frame is able to support forces. However, any small variation of the frame may instantly lead to instability. Maxwell realized that these conditions are elegantly expressed by the fact that Figure 3(b) constitutes an orthogonal projection of a closed polyhedron with planar faces. Remarkably, he proved in a constructive manner that any plane figure which may be regarded as an orthogonal projection of a three-dimensional polyhedron with planar faces admits a reciprocal and may therefore be identified with a frame which is in equilibrium. Indeed, let a plane figure be the orthogonal projection of a polyhedron and draw concurrent lines which are parallel to the normals to the faces of the polyhedron. The points of intersection with the plane on which the figure lies define another plane figure if we join by a line any two points which are associated with two adjacent faces. By construction, the lines of the second figure will be orthogonal to the corresponding lines of the original figure. Rotation by 90 degrees then produces a reciprocal figure. 

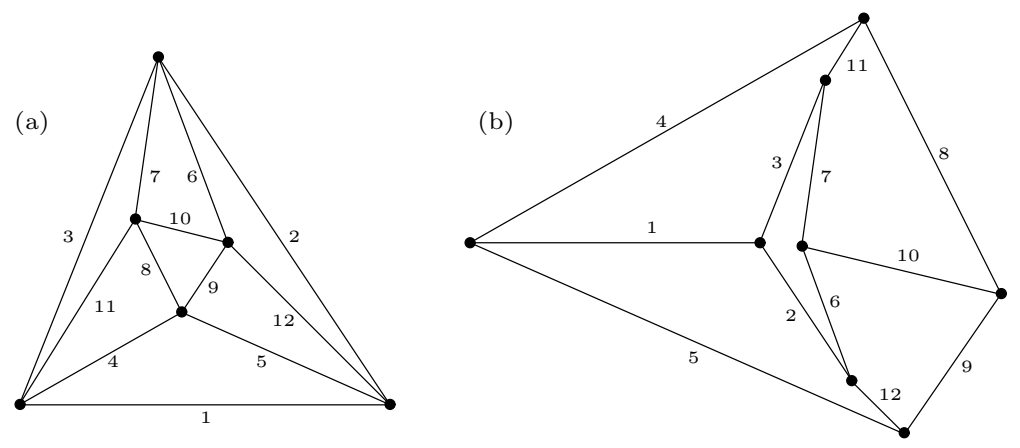

Figure 3: 'Indeterminate' or 'conditionally' reciprocal figures

The converse of Maxwell's theorem is not strictly true. It is not difficult to find plane figures which admit reciprocals but are not related to spatial polyhedra in the above-mentioned manner and frames which are in equilibrium but do not have (proper) reciprocal diagrams of forces. Nevertheless, if one makes the assumption that the combinatorics of the frame is that of a spherical polyhedron then the existence of a reciprocal, a spatial polyhedron and a selfstress (that is, a frame in equilibrium) are equivalent 30 .

In view of the following, it is now useful to return to the construction of the 'reciprocal triangles' displayed in Figure 1. Thus, given a figure of the type 11(a), we first draw three lines which are parallel to the edges $(1,4),(2,4)$ and $(3,4)$ and denote the points of intersection by 5,6 and 7 as shown in Figure 1(c). The two lines which pass through the points 5 and 7 and are parallel to the edges $(1,3)$ and $(2,3)$ respectively determine the point 8 . The existence of the reciprocal figure then guarantees that the line which passes through 5 and 8 is indeed parallel to the edge $(1,2)$. The phenomenom that during the construction of reciprocal figures certain lines turn out to be concurrent (cf. the construction of Figure $3(\mathrm{~b})$ ) provides the key to the link with integrable systems. Thus, in the following, we set in correspondence this geometric compatibility of reciprocal figures with the algebraic compatibility of linear systems defining discrete integrable systems.

\section{Reciprocal triangles, dilations and an 8-point relation}

In this section, we investigate in detail the geometric and algebraic properties of the simplest reciprocal figures, namely reciprocal triangles. In order to reveal connections with both inversive geometry and soliton theory, it proves convenient to complement Maxwell's geometric construction with a purely algebraic proof of the existence of reciprocal triangles. In fact, the proof given in [31] and recited below makes use of both a nonlinear algebraic system and its associated 

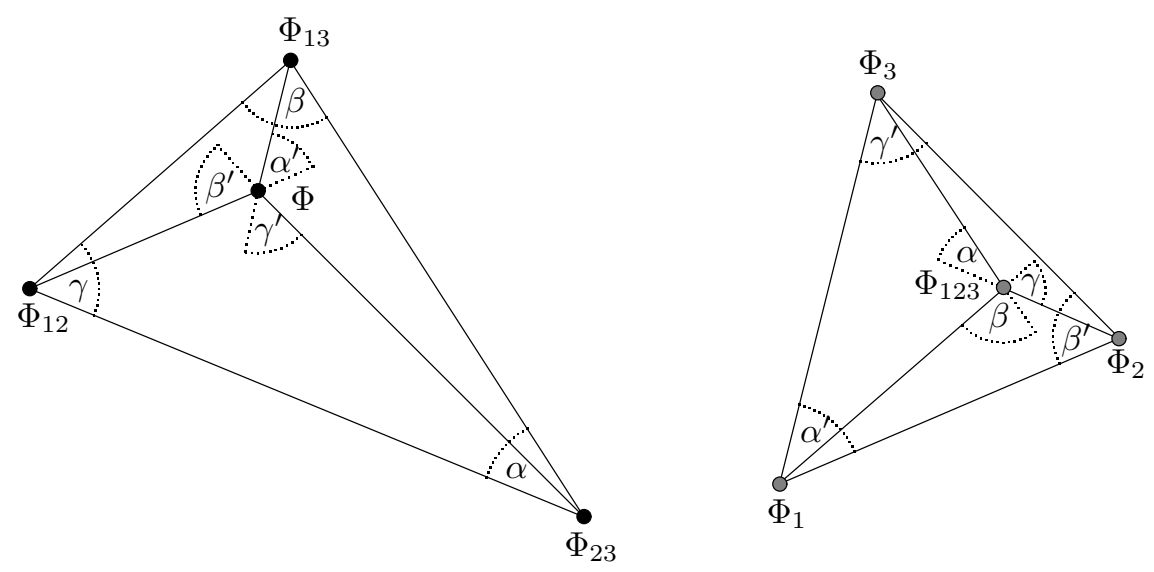

Figure 4: Reciprocal triangles

'linear representation'. Thus, consider a triangle $\Delta\left(\Phi_{23}, \Phi_{13}, \Phi_{12}\right)$ with vertices $\Phi_{23}, \Phi_{13}, \Phi_{12}$ and an additional point $\Phi$ on the plane. If $\Delta\left(\Phi_{1}, \Phi_{2}, \Phi_{3}\right)$ constitutes a triangle with edges $\left(\Phi_{1}, \Phi_{2}\right),\left(\Phi_{2}, \Phi_{3}\right),\left(\Phi_{3}, \Phi_{1}\right)$ being parallel to the line segments $\left(\Phi, \Phi_{12}\right),\left(\Phi, \Phi_{23}\right),\left(\Phi, \Phi_{13}\right)$ respectively then the two triangles are reciprocal if there exists a point $\Phi_{123}$ such that the line segments $\left(\Phi_{1}, \Phi_{123}\right)$, $\left(\Phi_{2}, \Phi_{123}\right),\left(\Phi_{3}, \Phi_{123}\right)$ are parallel to the edges $\left(\Phi_{13}, \Phi_{12}\right),\left(\Phi_{12}, \Phi_{23}\right),\left(\Phi_{23}, \Phi_{13}\right)$ respectively as indicated in Figure 4 . It turns out convenient to identify the plane with the complex plane and regard $\Phi, \ldots, \Phi_{123}$ as complex numbers. Accordingly, by assumption, there exist real dilation coefficients $a, b, c$ such that

$$
\begin{aligned}
& \Phi_{12}-\Phi=c\left(\Phi_{1}-\Phi_{2}\right) \\
& \Phi_{23}-\Phi=a\left(\Phi_{2}-\Phi_{3}\right) \\
& \Phi_{13}-\Phi=b\left(\Phi_{3}-\Phi_{1}\right)
\end{aligned}
$$

and the reciprocity condition amounts to the existence of a complex number $\Phi_{123} \in \mathbb{C}$ and three real dilations $a_{1}, b_{2}, c_{3} \in \mathbb{R}$ obeying the algebraic system

$$
\begin{aligned}
& \Phi_{123}-\Phi_{3}=c_{3}\left(\Phi_{13}-\Phi_{23}\right) \\
& \Phi_{123}-\Phi_{1}=a_{1}\left(\Phi_{12}-\Phi_{13}\right) \\
& \Phi_{123}-\Phi_{2}=b_{2}\left(\Phi_{23}-\Phi_{12}\right) .
\end{aligned}
$$

Now, any of the relations (3.2) may be regarded as a definition for $\Phi_{123}$ and elimination of $\Phi_{123}$ from (3.2) 1,2 and $(3.2)_{2,3}$ leads, on use of (3.1), to the necessary and sufficient conditions

$$
\begin{aligned}
& \left(1+c_{3} b+a_{1} b+c_{3} a\right)\left(\Phi_{1}-\Phi_{3}\right)=\left(c_{3} a-a_{1} c\right)\left(\Phi_{1}-\Phi_{2}\right) \\
& \left(1+a_{1} c+b_{2} c+a_{1} b\right)\left(\Phi_{2}-\Phi_{1}\right)=\left(a_{1} b-b_{2} a\right)\left(\Phi_{2}-\Phi_{3}\right) .
\end{aligned}
$$

\footnotetext{
${ }^{\dagger}$ It is noted that it is justified to use the term reciprocal triangles without referring to the points $\Phi$ and $\Phi_{123}$ since the latter are uniquely determined by the edges of the two triangles.
} 
Since the triangles are assumed to be non-degenerate, the real coefficients in (3.3) must vanish. If these four conditions on the dilations were independent then there would exist a constraint on the dilations $a, b, c$ and therefore the second triangle. However, Maxwell's geometric construction implies that this is not the case and, indeed, one condition turns out to be redundant. The remaining three conditions define the dilations $a_{1}, b_{2}, c_{3}$. Thus, the existence of the reciprocal triangle is guaranteed and, as a consequence, we may formulate the following theorem:

Theorem 1 (Algebraic description of reciprocal triangles).

If $\Delta\left(\Phi_{23}, \Phi_{13}, \Phi_{12}\right)$ and $\Delta\left(\Phi_{1}, \Phi_{2}, \Phi_{3}\right)$ constitute reciprocal triangles with 'interior points' $\Phi$ and $\Phi_{123}$ respectively then the dilations $a, b, c$ and $a_{1}, b_{2}, c_{3}$ as defined by (3.1) and (3.9) respectively are related by

$$
a_{1}=-\frac{a}{a b+b c+c a}, \quad b_{2}=-\frac{b}{a b+b c+c a}, \quad c_{3}=-\frac{c}{a b+b c+c a} .
$$

Conversely, let $\Phi_{1}, \Phi_{2}, \Phi_{3}$ and $\Phi$ be four generic points on the complex plane, $a, b, c \in \mathbb{R}$ be arbitrary non-vanishing real numbers and $a_{1}, b_{2}, c_{3}$ be given by (3.4). Then, the unique solution of the compatible linear system (3.1), (3.2) gives rise to the reciprocal triangles $\Delta\left(\Phi_{23}, \Phi_{13}, \Phi_{12}\right)$ and $\Delta\left(\Phi_{1}, \Phi_{2}, \Phi_{3}\right)$ with 'interior points' $\Phi$ and $\Phi_{123}$ respectively.

The second part of the above theorem expresses the fact that reciprocal triangles may be constructed in the following way: Given four points $\Phi_{1}, \Phi_{2}, \Phi_{3}$, $\Phi$ on the complex plane and three real numbers $a, b, c$, we draw three line segments $\left(\Phi, \Phi_{23}\right),\left(\Phi, \Phi_{13}\right),\left(\Phi, \Phi_{12}\right)$ which are parallel to the edges $\left(\Phi_{2}, \Phi_{3}\right)$, $\left(\Phi_{3}, \Phi_{1}\right),\left(\Phi_{1}, \Phi_{2}\right)$ and their lengths are determined by the dilations $a, b, c$ respectively. The lines which pass through the vertices $\Phi_{1}, \Phi_{2}, \Phi_{3}$ and are parallel to the line segments $\left(\Phi_{13}, \Phi_{12}\right),\left(\Phi_{12}, \Phi_{23}\right),\left(\Phi_{23}, \Phi_{13}\right)$ respectively then meet at a point $\Phi_{123}$ with associated dilations $a_{1}, b_{2}, c_{3}$ given by (3.4).

The relations (3.4) imply that, for instance, $c_{3} a=a_{1} c$ (cf. (3.3)). Hence, on expressing the dilations $a, c, a_{1}, c_{3}$ in terms of $\Phi, \ldots, \Phi_{123}$ by means of (3.1), (3.2) and rearranging terms, we are led to the following observation:

Corollary 1 (An 8-point relation). The quadruplets $\Phi_{1}, \Phi_{2}, \Phi_{3}, \Phi_{123}$ and $\Phi_{23}, \Phi_{13}, \Phi_{12}, \Phi$ associated with two reciprocal triangles satisfy the 8-point relation

$$
\frac{\left(\Phi_{1}-\Phi_{2}\right)\left(\Phi_{3}-\Phi_{123}\right)}{\left(\Phi_{2}-\Phi_{3}\right)\left(\Phi_{123}-\Phi_{1}\right)}=\frac{\left(\Phi_{23}-\Phi_{13}\right)\left(\Phi_{12}-\Phi\right)}{\left(\Phi_{13}-\Phi_{12}\right)\left(\Phi-\Phi_{23}\right)},
$$

that is

$$
Q\left(\Phi_{1}, \Phi_{2}, \Phi_{3}, \Phi_{123}\right)=Q\left(\Phi_{23}, \Phi_{13}, \Phi_{12}, \Phi\right),
$$

where the cross-ratio $Q$ of four points on the complex plane is defined as usual by

$$
Q\left(P_{1}, P_{2}, P_{3}, P_{4}\right)=\frac{\left(P_{1}-P_{2}\right)\left(P_{3}-P_{4}\right)}{\left(P_{2}-P_{3}\right)\left(P_{4}-P_{1}\right)} .
$$

It is noted that, by construction and due to the symmetries of the cross-ratio, the above 8-point relation is invariant under any simultaneous permutation of the arguments of the two cross-ratios. 


\section{Inversive geometry of reciprocal triangles}

In the preceding, it has been shown that reciprocal triangles provide particular solutions of the 8-point relation (3.5). This observation suggests that there should exist a complete geometric characterization of the 8-point relation in terms of reciprocal triangles if the latter are appropriately generalized. To this end, it is natural to adopt a wider definition of reciprocal figures (as suggested by Maxwell). Thus, two triangles are reciprocally related if corresponding pairs of edges are not necessarily parallel but meet at the same angle. In other words, relative rotations of the triangles are admissible and hence reciprocal triangles are characterized by the equality of corresponding angles as shown in Figure 4 .

Since the cross-ratio of four points on the complex plane is preserved by Möbius transformations

$$
\boldsymbol{\psi} \rightarrow \boldsymbol{\psi}^{\prime}=\frac{\tilde{a} \boldsymbol{\psi}+\tilde{b}}{\tilde{c} \boldsymbol{\psi}+\tilde{d}}
$$

with $\tilde{a} \tilde{d}-\tilde{b} \tilde{c} \neq 0$, it is evident that the 8-point relation is invariant under what may be referred to as local Möbius transformations acting independently on the quadruplets $\Phi_{1}, \Phi_{2}, \Phi_{3}, \Phi_{123}$ and $\Phi_{23}, \Phi_{13}, \Phi_{12}, \Phi$. Consequently, the 8-point relation is preserved by global Möbius transformations which act on the complex plane and therefore simultaneously on both quadruplets. In addition, the 8-point relation is seen to be invariant under complex conjugation. Thus, the 8-point relation turns out to be preserved by the group of inversive transformations which consists of Möbius transformations and complex conjugation and maps (generalized) circles to (generalized) circles [31, 32]. Particular inversive transformations include translations, scalings, rotations and inversions in a line or circle. The latter are represented by

$$
\boldsymbol{\psi} \rightarrow \boldsymbol{\psi}^{\prime}=\boldsymbol{\psi}_{*}+\frac{r^{2}}{\overline{\boldsymbol{\psi}}-\overline{\boldsymbol{\psi}}_{*}},
$$

where $r$ and $\boldsymbol{\psi}_{*}$ denote the radius and centre of a circle respectively. It is noted that inversive transformations may be decomposed into inversions. Accordingly, the 8-point relation constitutes an object of inversive geometry which, in the spirit of Klein, is concerned with those properties of figures on the plane which are preserved by inversive transformations [31]-33.

The above analysis of the symmetry group of the 8-point relation shows that a canonical definition of reciprocity is required to allow for the global group of inversive transformations and local group of Möbius transformations. It is therefore natural to adopt the following definition:

Definition 2 (Reciprocal $(4,6)$ configurations). A configuration consisting of four points on the complex plane which are linked by six circular arcs is termed a $(4,6)$ configuration if the circular extensions of the arcs meet at a point (cf. Figure 5). Two $(4,6)$ configurations are said to be reciprocally related if the six angles made by the circular arcs of one $(4,6)$ configuration equal those of the other $(4,6)$ configuration in the manner indicated in Figures 5 and 6 . 


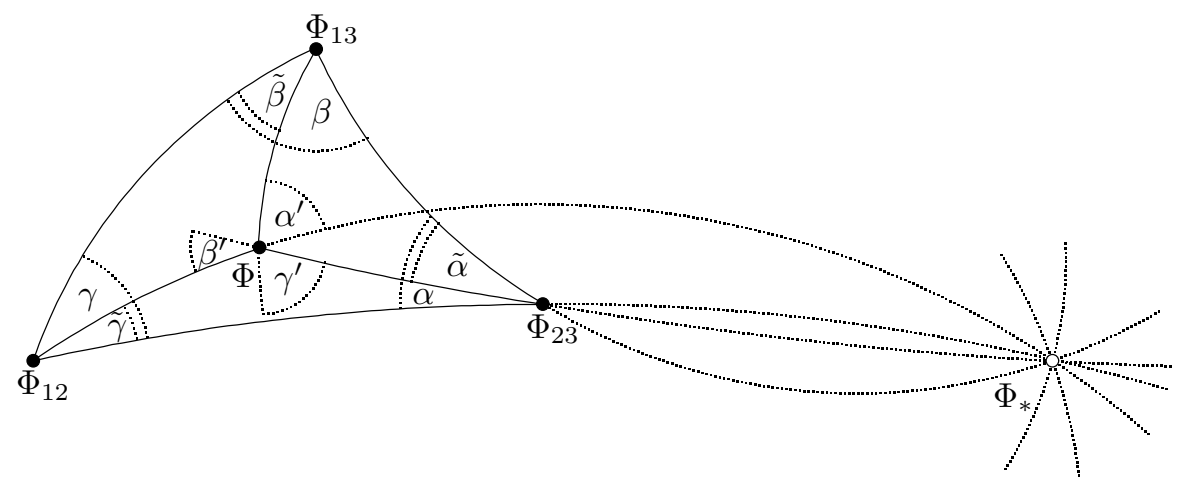

Figure 5: A $(4,6)$ configuration

It is evident that, by construction, $(4,6)$ configurations are images under inversive transformations of four points linked by six straight line segments. As a consequence, the angles in any of the four triangles made by three circular arcs add up to $\pi$. If the circular arcs of two reciprocal $(4,6)$ configurations degenerate to straight line segments then the usual definition of reciprocal triangles is retrieved. The generalization of Corollary 11 is now the following:

Theorem 2 (Inversive geometry of the 8-point relation). Two quadruplets $\left(\Phi_{1}, \Phi_{2}, \Phi_{3}, \Phi_{123}\right)$ and $\left(\Phi_{23}, \Phi_{13}, \Phi_{12}, \Phi\right)$ of complex numbers may be regarded as the vertices of two reciprocal $(4,6)$ configurations if and only if the 8-point relation

$$
Q\left(\Phi_{1}, \Phi_{2}, \Phi_{3}, \Phi_{123}\right)=Q\left(\Phi_{23}, \Phi_{13}, \Phi_{12}, \Phi\right)
$$

holds.

Proof. On the one hand, consider two reciprocal $(4,6)$ configurations with vertices $\Phi_{1}, \Phi_{2}, \Phi_{3}, \Phi_{123}$ and $\Phi_{23}, \Phi_{13}, \Phi_{12}, \Phi$ respectively. If we apply two local Möbius transformations which map the two points of intersection $\Phi_{*}$ and $\Phi_{*}^{\prime}$ to infinity (cf. Figures 司 and (6) then two reciprocal triangles are obtained. Since the cross-ratios associated with the two $(4,6)$ configurations are preserved by local Möbius transformations, Corollary 1 implies that the 8-point relation (4.3) is satisfied.

On the other hand, let the 8-point relation be satisfied and choose an arbitrary point $\Phi_{*}$. Then, by drawing circles through $\Phi_{*}$ and any two of the points $\Phi_{23}, \Phi_{13}, \Phi_{12}, \Phi$, a $(4,6)$ configuration is obtained. The circles passing through the point $\Phi$ meet at some angles $\alpha^{\prime}, \beta^{\prime}$ and $\gamma^{\prime}$ as indicated in Figure 5 . Here, we regard angles as oriented quantities. Another $(4,6)$ configuration is now constructed in the following way: If $\lambda, \mu, \nu$ denote the angles made by the line segments $\left(\Phi_{1}, \Phi_{2}\right),\left(\Phi_{2}, \Phi_{3}\right),\left(\Phi_{3}, \Phi_{1}\right)$ then we may draw three circles which pass through the pairs $\left\{\Phi_{2}, \Phi_{3}\right\},\left\{\Phi_{3}, \Phi_{1}\right\},\left\{\Phi_{1}, \Phi_{2}\right\}$ and meet these line segments at 


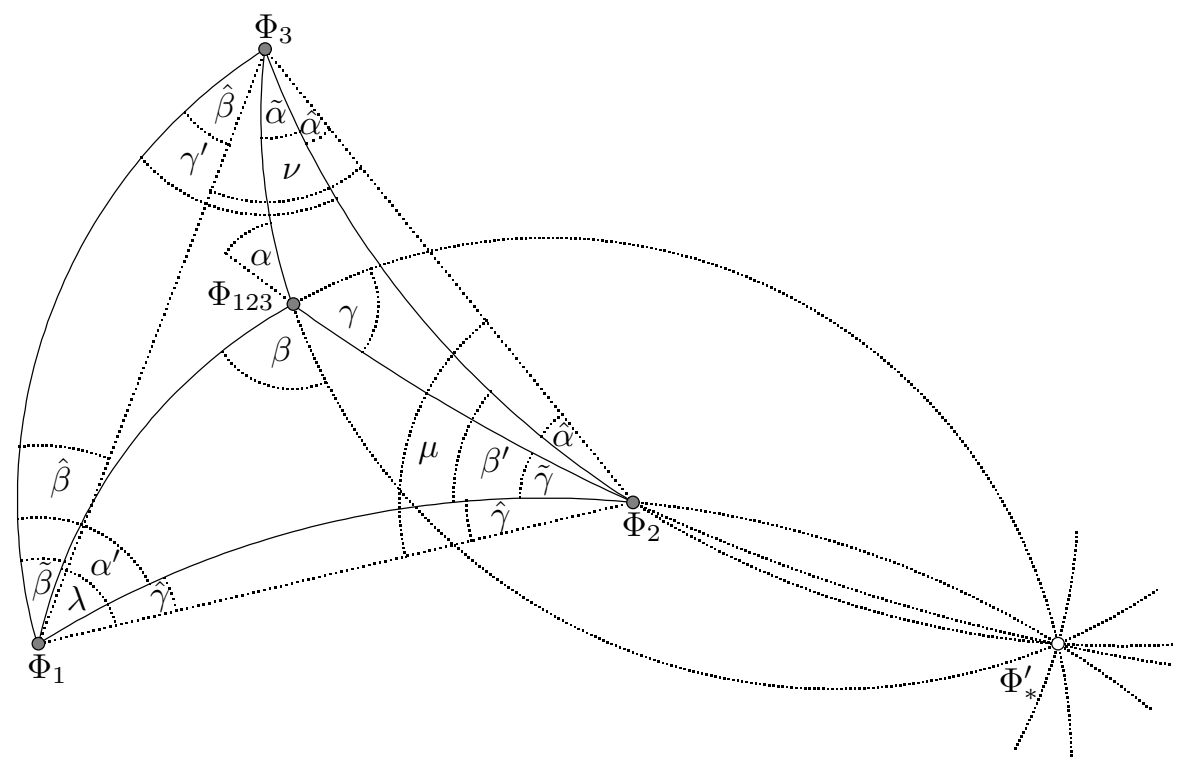

Figure 6: A reciprocal $(4,6)$ configuration

angles $\hat{\alpha}=\alpha^{\prime}-\lambda, \hat{\beta}=\beta^{\prime}-\mu, \hat{\gamma}=\gamma^{\prime}-\nu$ as shown in Figure 6. By construction, these circles meet at angles $\alpha^{\prime}, \beta^{\prime}, \gamma^{\prime}$. Since $\alpha^{\prime}+\beta^{\prime}+\gamma^{\prime}=\pi$, the three circles intersect at a point, $\Phi_{*}^{\prime}$, say. Finally, another three circles $S_{1}, S_{2}, S_{3}$ are defined by the requirement that they pass through the pairs $\left\{\Phi_{1}, \Phi_{*}^{\prime}\right\},\left\{\Phi_{2}, \Phi_{*}^{\prime}\right\}$, $\left\{\Phi_{3}, \Phi_{*}^{\prime}\right\}$ and meet the other three circles at angles $\tilde{\alpha}, \tilde{\beta}, \tilde{\gamma}$ which are defined in Figure . We now apply two local Möbius transformations which map the points $\Phi_{*}$ and $\Phi_{*}^{\prime}$ to infinity and the twelve circles to twelve straight lines. The existence theorem of reciprocal triangles then implies that the images of the circles $S_{1}, S_{2}, S_{3}$ are concurrent and hence the circles $S_{1}, S_{2}, S_{3}$ intersect at a point $\Phi_{\circ \circ \circ}$, say. Thus, the quadruplets $\left(\Phi_{1}, \Phi_{2}, \Phi_{3}, \Phi_{\circ \circ \circ}\right)$ and $\left(\Phi_{23}, \Phi_{13}, \Phi_{12}, \Phi\right)$ of points constitute the vertices of two reciprocal $(4,6)$ configurations and hence

$$
Q\left(\Phi_{1}, \Phi_{2}, \Phi_{3}, \Phi_{\circ \circ \circ}\right)=Q\left(\Phi_{23}, \Phi_{13}, \Phi_{12}, \Phi\right) .
$$

However, since, by assumption, the 8-point relation (4.3) is likewise satisfied, it is concluded that $\Phi_{\circ \circ \circ}=\Phi_{123}$. This completes the proof.

Another algebraic description of reciprocal $(4,6)$ configurations is obtained by introducing complex dilations $a, b, c$ and $a_{1}, b_{2}, c_{3}$ according to

$$
\begin{aligned}
& \Phi_{12}-\Phi=c\left(\Phi_{1}-\Phi_{2}\right) \\
& \Phi_{23}-\Phi=a\left(\Phi_{2}-\Phi_{3}\right) \\
& \Phi_{13}-\Phi=b\left(\Phi_{3}-\Phi_{1}\right)
\end{aligned}
$$


and

$$
\begin{aligned}
& \Phi_{123}-\Phi_{3}=c_{3}\left(\Phi_{13}-\Phi_{23}\right) \\
& \Phi_{123}-\Phi_{1}=a_{1}\left(\Phi_{12}-\Phi_{13}\right) \\
& \Phi_{123}-\Phi_{2}=b_{2}\left(\Phi_{23}-\Phi_{12}\right) .
\end{aligned}
$$

As in the case of reciprocal triangles, the latter system implies that

$$
\begin{aligned}
& \left(1+c_{3} b+a_{1} b+c_{3} a\right)\left(\Phi_{1}-\Phi_{3}\right)=\left(c_{3} a-a_{1} c\right)\left(\Phi_{1}-\Phi_{2}\right) \\
& \left(1+a_{1} c+b_{2} c+a_{1} b\right)\left(\Phi_{2}-\Phi_{1}\right)=\left(a_{1} b-b_{2} a\right)\left(\Phi_{2}-\Phi_{3}\right) .
\end{aligned}
$$

However, the 8-point relation (4.3) is equivalent to any of the relations $a_{1} b=b_{2} a$, $b_{2} c=c_{3} b$ or $c_{3} a=a_{1} c$. Consequently, the right-hand sides of (4.7) vanish and the left-hand sides provide another two constraints on the complex dilations. Thus, we obtain the following generalization of Theorem 1:

Theorem 3 (Algebraic description of reciprocal $(4,6)$ configurations). The complex dilations $a, b, c$ and $a_{1}, b_{2}, c_{3}$ as defined by (4.5) and (4.6) associated with two reciprocal $(4,6)$ configurations are related by

$$
a_{1}=-\frac{a}{a b+b c+c a}, \quad b_{2}=-\frac{b}{a b+b c+c a}, \quad c_{3}=-\frac{c}{a b+b c+c a} .
$$

Conversely, let $\Phi_{1}, \Phi_{2}, \Phi_{3}$ and $\Phi$ be four generic points on the complex plane, $a, b, c \in \mathbb{C}$ be arbitrary non-vanishing complex numbers and $a_{1}, b_{2}, c_{3}$ be given by (4.8). Then, the unique solution of the compatible linear system (4.5), (4.8) gives rise to reciprocal $(4,6)$ configurations with vertices $\Phi_{1}, \Phi_{2}, \Phi_{3}, \Phi_{123}$ and $\Phi_{23}, \Phi_{13}, \Phi_{12}, \Phi$ respectively.

Theorems 1 and 3 are formulated in such a way that a remarkable connection with soliton theory is readily established. Indeed, it is shown below that the nonlinear system (4.8) may be identified with a well-known and distinct discrete integrable equation with (4.5) being its standard linear representation. A similar geometric link with the Schwarzian Kadomtsev-Petviashvili hierarchy provided by the ancient Greek Theorem of Menelaus has been recorded earlier in [28].

\section{The discrete BKP equation}

In this section, we are concerned with (pairs) of lattices which consist of an infinite number of reciprocal triangles or $(4,6)$ configurations. In order to show the existence of such lattices, it is convenient to introduce a canonical notation associated with three-dimensional lattices on the complex plane of $\mathbb{Z}^{3}$ combinatorics, that is maps of the form

$$
\Phi: \mathbb{Z}^{3} \rightarrow \mathbb{C}, \quad\left(n_{1}, n_{2}, n_{3}\right) \mapsto \Phi\left(n_{2}, n_{2}, n_{3}\right) .
$$



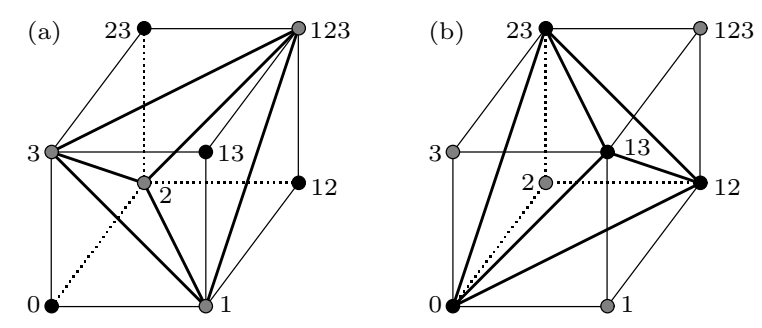

Figure 7: The elementary cells of $G^{(1)}$ and $G^{(0)}$

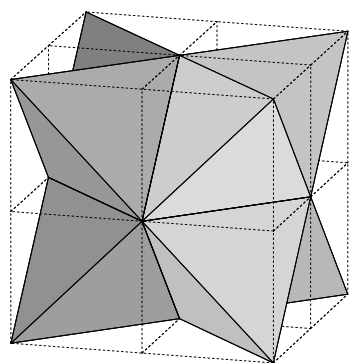

Figure 8: The stella octangula

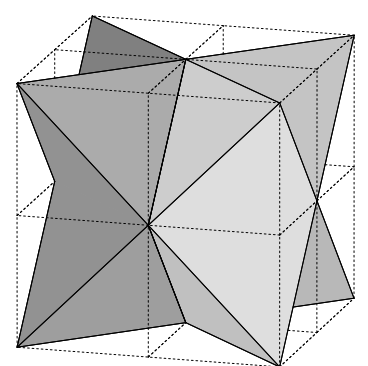

Figure 9: A modified stella octangula

Thus, we supress the arguments of $\Phi$ and indicate unit increments of the discrete variables or, equivalently, shifts on the lattice along the edges by indices:

$$
\Phi=\Phi\left(n_{1}, n_{2}, n_{3}\right), \quad \Phi_{1}=\Phi\left(n_{1}+1, n_{2}, n_{3}\right), \quad \Phi_{12}=\Phi\left(n_{1}+1, n_{2}+1, n_{3}\right) \ldots
$$

Since reciprocal triangles constitute projections of tetrahedral polyhedra, it is natural to consider lattices on the complex plane which represent images of three-dimensional lattices consisting of tetrahedra. A canonical construction of the latter is obtained by starting at a vertex of the $\mathbb{Z}^{3}$ cubic lattice and drawing diagonals across the faces of the cubes. The six diagonals on each cube then form a tetrahedron as shown in Figure 7, while the polyhedron inscribed in eight adjacent cubes is readily seen to be the stellated octahedron (stella octangula), that is an octahedron which is enclosed by eight tetrahedra (cf. Figure 8). Accordingly, the complete lattice is obtained by stacking stella octangulae. This lattice may be interpreted as a face-centred cubic (fcc) lattice or a face-centred cubic sphere packing [34] with the edges linking the centres of the spheres. Hence, we here consider lattices on the complex plane of the form

$$
\begin{aligned}
\Phi^{(0)}: G^{(0)} & \rightarrow \mathbb{C} \\
G^{(0)} & =\left\{\left(n_{1}, n_{2}, n_{3}\right) \in \mathbb{Z}^{3}: n_{1}+n_{2}+n_{3} \text { even }\right\} .
\end{aligned}
$$


It is our aim to show that two lattices of this type which consist of reciprocal triangles or $(4,6)$ configurations are integrable. To this end, the second lattice of the type (5.3) is labelled according to (cf. Figure 7(a))

$$
\begin{aligned}
\Phi^{(1)}: G^{(1)} & \rightarrow \mathbb{C} \\
G^{(1)} & =\left\{\left(n_{1}, n_{2}, n_{3}\right) \in \mathbb{Z}^{3}: n_{1}+n_{2}+n_{3} \text { odd }\right\} .
\end{aligned}
$$

Since the set $G^{(1)}$ is the complement of $G^{(0)}$ with respect to $\mathbb{Z}^{3}$, we are now in a position to combine the lattices $\Phi^{(0)}$ and $\Phi^{(1)}$ to one lattice $\Phi$ :

$$
\Phi(\boldsymbol{n})=\Phi^{(i)}(\boldsymbol{n}) \text { if } \boldsymbol{n} \in G^{(i)} .
$$

This is merely done for reasons of book-keeping. It is interesting to note that the structure of these interpenetrating fcc lattices is precisely that of the sodium chloride crystal, where the $\mathrm{Na}^{+}$ions form one fcc lattice and the second fcc lattice consists of $\mathrm{Cl}^{-}$ions.

It is now evident that the lattices $\Phi^{(0)}$ and $\Phi^{(1)}$ are composed of reciprocal triangles or $(4,6)$ configurations if the lattice $\Phi$ obeys the linear lattice equations

$$
\begin{aligned}
& \Phi_{12}-\Phi=c\left(\Phi_{1}-\Phi_{2}\right) \\
& \Phi_{23}-\Phi=a\left(\Phi_{2}-\Phi_{3}\right) \\
& \Phi_{13}-\Phi=b\left(\Phi_{3}-\Phi_{1}\right),
\end{aligned}
$$

where $a, b, c$ are as yet unspecified dilation functions. For such lattices to exist, the compatibility conditions $\left(\Phi_{12}\right)_{3}=\left(\Phi_{23}\right)_{1}=\left(\Phi_{13}\right)_{2}$ need to be satisfied. These encapsulate the fact that the construction of the vertex $\Phi_{123}$ in the three different ways

$$
\begin{aligned}
& \Phi_{123}-\Phi_{3}=c_{3}\left(\Phi_{13}-\Phi_{23}\right) \\
& \Phi_{123}-\Phi_{1}=a_{1}\left(\Phi_{12}-\Phi_{13}\right) \\
& \Phi_{123}-\Phi_{2}=b_{2}\left(\Phi_{23}-\Phi_{12}\right)
\end{aligned}
$$

must lead to the same result. Since the systems $(4.6)$ and $(5.7)$ are identical, the compatibility conditions coincide with those associated with reciprocal triangles or $(4,6)$ configurations, that is

$$
E^{1}\left(\Phi_{1}-\Phi_{3}\right)=E^{2}\left(\Phi_{1}-\Phi_{2}\right), \quad E^{3}\left(\Phi_{2}-\Phi_{3}\right)=E^{4}\left(\Phi_{2}-\Phi_{3}\right)
$$

with the coefficients $E^{i}$ given by

$$
\begin{array}{ll}
E^{1}=1+c_{3} b+a_{1} b+c_{3} a, & E^{2}=c_{3} a-a_{1} c \\
E^{3}=1+a_{1} c+b_{2} c+a_{1} b, & E^{4}=a_{1} b-b_{2} a .
\end{array}
$$

Moreover, the results of Section 3 imply that the coefficients $E^{i}$ vanish if and only if the dilation functions satisfy the algebraic system (4.8) now regarded as lattice equations. Any real or complex solution of the latter therefore defines lattices composed of reciprocal triangles or $(4,6)$ configurations respectively. 
The fact that the nonlinear system of difference equations 4.8 is obtainable from the compatibility conditions for the linear system (5.6) suggests that there exists a connection with soliton theory. Indeed, the relations $a_{1} b=b_{2} a$, $b_{2} c=c_{3} b$ and $c_{3} a=a_{1} c$ guarantee that there exists a potential $\tau$ which parametrizes the dilations $a, b, c$ according to

$$
a=\frac{\tau_{2} \tau_{3}}{\tau \tau_{23}}, \quad b=\frac{\tau_{1} \tau_{3}}{\tau \tau_{13}}, \quad c=\frac{\tau_{1} \tau_{2}}{\tau \tau_{12}} .
$$

The system (4.8) then reduces to an integrable single equation known as the discrete BKP (dBKP) equation 21] and (5.6) is nothing but its standard linear representation (see, e.g., 22]). Thus, the following theorem obtains:

Theorem 4 (BKP lattices). Three-dimensional lattices $\Phi$ on the complex plane with constituent sublattices $\Phi^{(0)}$ and $\Phi^{(1)}$ of fcc combinatorics consist of reciprocal $(4,6)$ configurations (triangles) with corresponding quadruplets of vertices $\left(\Phi_{23}, \Phi_{13}, \Phi_{12}, \Phi\right)$ and $\left(\Phi_{1}, \Phi_{2}, \Phi_{3}, \Phi_{123}\right)$ if and only if the complex (real) dilations defined by

$$
\begin{aligned}
& \Phi_{12}-\Phi=\frac{\tau_{1} \tau_{2}}{\tau \tau_{12}}\left(\Phi_{1}-\Phi_{2}\right) \\
& \Phi_{23}-\Phi=\frac{\tau_{2} \tau_{3}}{\tau \tau_{23}}\left(\Phi_{2}-\Phi_{3}\right) \\
& \Phi_{13}-\Phi=\frac{\tau_{1} \tau_{3}}{\tau \tau_{13}}\left(\Phi_{3}-\Phi_{1}\right)
\end{aligned}
$$

may be parametrized in terms of solutions of the discrete BKP equation

$$
\tau \tau_{123}+\tau_{1} \tau_{23}+\tau_{2} \tau_{13}+\tau_{3} \tau_{12}=0
$$

Lattices of this type are equivalently described by the 8-point lattice equation

$$
Q\left(\Phi_{1}, \Phi_{2}, \Phi_{3}, \Phi_{123}\right)=Q\left(\Phi_{23}, \Phi_{13}, \Phi_{12}, \Phi\right) .
$$

The celebrated Kadomtsev-Petviashvili (KP) hierarchy of integrable equations is often referred to as the AKP hierarchy due to its deep connection with the Lie algebra $A_{\infty}=g l(\infty)$ 35. The hierarchy associated with the Lie algebra $B_{\infty}=s o(\infty)$ is known as the BKP hierarchy. The dBKP equation encodes the complete BKP hierarchy of soliton equations [21]. Indeed, by applying appropriate continuum limits to the dBKP equation, any member of the BKP family may be obtained. Proto-typical examples are the 2+1-dimensional Sawada-Kotera and Nizhnik-Veselov-Novikov equations [35].

It was pointed out in 22] that there exists a 'natural' continuum limit in which the variables $n_{i}$ are regarded as direct discretizations of some continuous variables which leads to the integrable $2+1$-dimensional generalization of the classical sine-Gordon equation set down in [36]. In fact, the dBKP equation was shown to represent a superposition principle for eight solutions of the 2+1-dimensional sine-Gordon system generated by the classical Moutard transformation [37]. In subsequent work [23], the superposition principle for the 
associated eigenfunctions was set down but it did not occur to the authors that it may be cast into the form of the 8-point relation (5.13). By construction, the latter is equivalent to the dSKP equation. In analogy with the discrete (Schwarzian) KP equation [38]-40], it may be termed discrete Schwarzian BKP (dSBKP) equation since it is invariant under Möbius transformations and constitutes a compact form of the hierarchy of singular manifold equations associated with the BKP hierarchy.

In summary, it has been shown that, in the setting of inversive geometry, reciprocal triangles and, more generally, reciprocal $(4,6)$ configurations encapsulate the integrable dBKP and dSBKP equations and hence the associated semi-discrete and continuous hierarchies of integrable equations. It is therefore natural to refer to the lattices composed of these figures as BKP lattices. BKP lattices consisting of reciprocal triangles correspond to real solutions of the BKP equation. Purely imaginary solutions of the BKP equation in the form (4.8) may be associated with lattices that contain reciprocal triangles whose edges meet at right angles. We observe in passing that BKP lattices consisting of reciprocal triangles may also be defined in ambient spaces of arbitrary dimension. Indeed, if we regard $\Phi$ as a real vector-valued function in $\mathbb{R}^{n}$ and the function $\tau$ constitutes a real solution of the BKP equation then the linear system (5.11) remains compatible and the corresponding lattice is composed of reciprocal triangles. Furthermore, since both the vertices $\Phi_{1}, \Phi_{2}, \Phi_{3}, \Phi_{123}$ and the vertices $\Phi_{23}, \Phi_{13}, \Phi_{12}, \Phi$ are coplanar, the quadrilaterals $\left(\Phi, \Phi_{i}, \Phi_{k}, \Phi_{i k}\right), i<k$ are planar (but non-embedded). Thus, lattices in $\mathbb{R}^{n}$ consisting of reciprocal triangles constitute particular conjugate lattices [41]. From an algebraic point of view, this is evident since (5.11) represents three coupled discrete Darboux equations [42, 43].

\section{Particular BKP lattices. Sine-Gordon and Tzitzéica lattices}

There exists a great variety of reductions of both the dBKP and dSBKP equations each of which may now be analysed with respect to its geometric significance. Here, we embark on such a geometric analysis and discuss two canonical reductions leading to particular BKP lattices. Firstly, since the dSBKP equation is invariant under inversion, it is natural to focus on lattices which constitute fixed points of inversive transformations. For instance, if we consider the reduction $\Phi=1 / \bar{\Phi}$ then the parametrization

$$
\Phi=e^{2 \mathrm{i} \omega}
$$

where $\omega$ denotes a real function, reduces the dSBKP equation to

$$
\frac{\sin \left(\omega_{1}-\omega_{2}\right) \sin \left(\omega_{3}-\omega_{123}\right)}{\sin \left(\omega_{2}-\omega_{3}\right) \sin \left(\omega_{123}-\omega_{1}\right)}=\frac{\sin \left(\omega_{23}-\omega_{13}\right) \sin \left(\omega_{12}-\omega\right)}{\sin \left(\omega_{13}-\omega_{12}\right) \sin \left(\omega-\omega_{23}\right)}
$$


As shown in [23], this equation constitutes an integrable discretization of the 2+1-dimensional sine-Gordon equation

$$
\omega_{x y z}=\omega_{x} \omega_{y z} \cot \omega-\omega_{y} \omega_{x z} \tan \omega
$$

which was first set down by Darboux [4] in connection with triply orthogonal systems of surfaces and later rediscovered in [36, 45] as a canonical reduction of the 2+1-dimensional sine-Gordon system alluded to in the preceding section. It has also been derived in the context of three-dimensional discrete 'curvature' lattices on the plane 46 .

Secondly, it is natural to consider BKP lattices which are mapped to itself by discrete rotations, scalings or both. For instance, it may be assumed that $k$ shifts along the edges in the $n_{3}$-direction amount to a combination of a scaling and a rotation of the lattice, viz

$$
\Phi\left(n_{3}+k\right)=c \Phi\left(n_{3}\right), \quad c \in \mathbb{C}, k \in \mathbb{N} .
$$

This corresponds to $k$-periodic reductions of the dBKP equation. It turns out that the dBKP equation may be solved explicitly if $k=1$ and reduces to a linear equation if the period is $k=2$. The first nontrivial case is given by $k=3$, that is

$$
\Phi_{333}=c \Phi, \quad \tau_{333}=\tau .
$$

Thus, if we introduce the quantities

$$
\rho=\tau_{3}, \quad \sigma=\tau_{33}
$$

then the dBKP equation (5.12) turns into the coupled system of three twodimensional equations

$$
\begin{array}{r}
\tau \rho_{12}+\tau_{1} \rho_{2}+\tau_{2} \rho_{1}+\rho \tau_{12}=0 \\
\rho \sigma_{12}+\rho_{1} \sigma_{2}+\rho_{2} \sigma_{1}+\sigma \rho_{12}=0 \\
\sigma \tau_{12}+\sigma_{1} \tau_{2}+\sigma_{2} \tau_{1}+\tau \sigma_{12}=0 .
\end{array}
$$

Inspection of this system shows that it is convenient to define quantities $\phi, \psi$ and $H$ according to

$$
\rho=\tau \phi, \quad \sigma=\tau \psi, \quad H=-\frac{\tau_{1} \tau_{2}}{\tau \tau_{12}}
$$

so that we obtain the two linear equations

$$
\phi_{12}+\phi=H\left(\phi_{1}+\phi_{2}\right), \quad \psi_{12}+\psi=H\left(\psi_{1}+\psi_{2}\right)
$$

subject to the quadratic constraint

$$
H\left[\psi\left(\phi_{1}+\phi_{2}\right)+\left(\psi_{1}+\psi_{2}\right) \phi-\psi_{2} \phi_{1}-\psi_{1} \phi_{2}\right]=2 \psi \phi .
$$


If we shift this constraint in the $n_{1}$ - and $n_{2}$-directions then we obtain two equations which may be separated into four linear equations by introducing functions of separation $A$ and $B$. These read

$$
\begin{aligned}
\phi_{11}-\phi_{1} & =\frac{H_{1}-1}{H_{1}(H-1)}\left(\phi_{1}-\phi\right)+\frac{A}{H-1}\left(\phi_{12}-\phi_{1}\right) \\
\phi_{22}-\phi_{2} & =\frac{H_{2}-1}{H_{2}(H-1)}\left(\phi_{2}-\phi\right)+\frac{B}{H-1}\left(\phi_{12}-\phi_{2}\right) \\
\psi_{11}-\psi_{1} & =\frac{H_{1}-1}{H_{1}(H-1)}\left(\psi_{1}-\psi\right)-\frac{A}{H-1}\left(\psi_{12}-\psi_{1}\right) \\
\psi_{22}-\psi_{2} & =\frac{H_{2}-1}{H_{2}(H-1)}\left(\psi_{2}-\psi\right)-\frac{B}{H-1}\left(\psi_{12}-\psi_{2}\right) .
\end{aligned}
$$

We observe that the two systems $(6.11)_{1,2}$ and $(6.11)_{3,4}$ differ only by the signs in front of $A$ and $B$. In fact, the two systems may be regarded as mutually adjoint with $(6.10)$ being an admissible constraint. This is reflected by the fact that the compatibility conditions $\left(\phi_{11}\right)_{2}=\left(\phi_{12}\right)_{1},\left(\phi_{22}\right)_{1}=\left(\phi_{12}\right)_{2}$ and $\left(\psi_{11}\right)_{2}=\left(\psi_{12}\right)_{1},\left(\psi_{22}\right)_{1}=\left(\psi_{12}\right)_{2}$ produce the same nonlinear difference equations for $A, B$ and $H$, namely

$$
\begin{gathered}
A_{2}=\frac{H_{1}}{H} A, \quad B_{1}=\frac{H_{2}}{H} B \\
H_{12}=\frac{H(H-1)}{H^{2}\left(H_{1}+H_{2}-H_{1} H_{2}\right)-H+A B H_{1} H_{2}} .
\end{gathered}
$$

The latter system constitutes an integrable discretization of the gauge-invariant form of the classical Tzitzéica equation 47, 48

$$
(\ln h)_{x y}=h-h^{-2} \text {. }
$$

It is interesting to note that the discrete Tzitzéica system has been shown to govern canonical discrete analogues of affine spheres which have been defined in a purely geometric manner in [26, 27].

A single equation may be obtained by parametrizing $A, B$ and $H$ according to

$$
A=\frac{\tilde{\tau}_{1}^{2}}{\tilde{\tau} \tilde{\tau}_{11}}, \quad H=\frac{\tilde{\tau}_{1} \tilde{\tau}_{2}}{\tilde{\tau} \tilde{\tau}_{12}}, \quad B=\frac{\tilde{\tau}_{2}^{2}}{\tilde{\tau} \tilde{\tau}_{22}}
$$

so that 6.12$)_{1,2}$ are identically satisfied. The remaining relation then reduces to the discrete Tzitzéica equation

$$
\left|\begin{array}{ccc}
\tilde{\tau} & \tilde{\tau}_{1} & \tilde{\tau}_{11} \\
\tilde{\tau}_{2} & \tilde{\tau}_{12} & \tilde{\tau}_{112} \\
\tilde{\tau}_{22} & \tilde{\tau}_{122} & \tilde{\tau}_{1122}
\end{array}\right|+\tilde{\tau}_{12}^{3}=0
$$

Since $\tilde{\tau}=(-1)^{n_{1} n_{2}} \tau$ without loss of generality, it is evident that the quantities $\tilde{\rho}=(-1)^{n_{1} n_{2}} \rho$ and $\tilde{\sigma}=(-1)^{n_{1} n_{2}} \sigma$ constitute another two solutions of the 
discrete Tzitzéica equation. In fact, the relations $(6.8)_{1,2}$ imply that these three solutions of the Tzitzéica equation are related by the discrete version of the classical Tzitzéica transformation set down in 25, 26.

The above link with the discrete Tzitzéica transformation may be further investigated by considering the linear system (5.11) for the lattice function $\Phi$. Thus, if we introduce the notation

$$
\Phi_{3}=\boldsymbol{\psi}, \quad \Phi_{33}=\Xi
$$

then we obtain nine equations with coefficients depending on $\tau, \rho$ and $\sigma$. For brevity, we merely state that this system may be decoupled into

$$
\begin{aligned}
\Phi_{11}-\Phi_{1} & =\frac{H_{1}-1}{H_{1}(H-1)}\left(\Phi_{1}-\Phi\right)-\lambda \frac{A}{H-1}\left(\Phi_{12}+\Phi_{1}\right) \\
\Phi_{12}-\Phi & =-H\left(\Phi_{1}-\Phi_{2}\right) \\
\Phi_{22}+\Phi_{2} & =-\frac{H_{2}-1}{H_{2}(H-1)}\left(\Phi_{2}+\Phi\right)-\frac{1}{\lambda} \frac{B}{H-1}\left(\Phi_{12}-\Phi_{2}\right),
\end{aligned}
$$

where $\lambda=(1-c) /(1+c)$, and

$$
\begin{aligned}
& \boldsymbol{\psi}=c\left\{\Phi+\frac{H}{(\lambda-1)(H-1) \phi}\left[\lambda\left(\phi_{1}-\phi\right)\left(\Phi_{2}+\Phi\right)+\left(\phi_{2}-\phi\right)\left(\Phi_{1}-\Phi\right)\right]\right\} \\
& \Xi=\Phi+\frac{H}{(\lambda+1)(H-1) \psi}\left[\lambda\left(\psi_{1}-\psi\right)\left(\Phi_{2}+\Phi\right)-\left(\psi_{2}-\psi\right)\left(\Phi_{1}-\Phi\right)\right] .
\end{aligned}
$$

The substitution $\Phi \rightarrow(-1)^{n_{2}} \Phi$ shows that $(6.17)$ is nothing but another copy of the linear representation $(6.9)_{1},(6.11)_{1,2}$ of the discrete Tzitzéica system if the invariance $A \rightarrow \lambda A, B \rightarrow B / \lambda$ of (6.12) is taken into account. Hence, the system (6.17) represents the standard parameter-dependent linear representation of the discrete Tzitzéica system 25]-27]. Furthermore, for symmetry reasons, the quantities $\psi$ and $\Xi$ obey analogous linear systems associated with the solutions $\tilde{\rho}$ and $\tilde{\sigma}$ of the discrete Tzitzéica equation. Indeed, it turns out that $\boldsymbol{\psi}$ and $\Xi$ as given by (6.18) are discrete Tzitzéica transforms [25, 26] of $\Phi$. We have therefore established that BKP lattices subject to the symmetry constraint $\Phi_{333}=c \Phi$ may be decomposed into two-dimensional 'Tzitzéica' lattices defined by (6.17) and their discrete Tzitzéica transforms $\psi$ and $\Xi$.

We conclude this section with the remark that the dBKP equation may also be regarded as a fully discrete version of the $B_{\infty}$ Toda lattice. Accordingly, the discrete Tzitzéica equation constitutes the period 3 reduction of the fully discrete Toda lattice. This is in harmony with the fact that the Tzitzéica equation not only appears in the context of affine differential geometry [49] but also constitutes the period 3 reduction of the $B_{\infty}$ Toda lattice [50].

\section{Continuum limits. Quasi-conformal mappings}

As mentioned earlier, any member of the (S)BKP hierarchy may be retrieved from the d(S)BKP equation by application of an appropriate continuum limit. 
Furthermore, if we make the substitution (6.1), where the function $\omega$ is now regarded to be complex, then the dSBKP equation assumes the trigonometric form (6.2). The connection with Darboux's (complex) sine-Gordon-type equation (6.3) is established by relabelling the lattice according to

$$
\omega \rightarrow \frac{(-1)^{n_{3}}}{2} \omega+n_{2} \frac{\pi}{2}
$$

leading to

$$
\frac{\cos \left[\frac{1}{2}\left(\omega_{1}-\omega_{2}\right)\right] \cos \left[\frac{1}{2}\left(\omega_{3}-\omega_{123}\right)\right]}{\cos \left[\frac{1}{2}\left(\omega_{2}+\omega_{3}\right)\right] \cos \left[\frac{1}{2}\left(\omega_{123}+\omega_{1}\right)\right]}=\frac{\cos \left[\frac{1}{2}\left(\omega_{23}-\omega_{13}\right)\right] \cos \left[\frac{1}{2}\left(\omega_{12}-\omega\right)\right]}{\cos \left[\frac{1}{2}\left(\omega_{13}+\omega_{12}\right)\right] \cos \left[\frac{1}{2}\left(\omega+\omega_{23}\right)\right]},
$$

and then performing the limit

$$
x=\epsilon n_{1}, \quad y=\epsilon n_{2}, \quad z=\epsilon n_{3}, \quad \epsilon \rightarrow 0 .
$$

An equation of second order is obtained by considering the natural continuum limit in which the polygons $\Phi\left(n_{i}=\right.$ const, $n_{k}=$ const $)$ become coordinate lines on the complex plane. Thus, if we set

$$
\Phi_{i}=\Phi+\epsilon \Phi_{x_{i}}+O\left(\epsilon^{2}\right), \quad i=1,2,3,
$$

where $\epsilon$ denotes a lattice parameter and $\Phi_{x_{i}}=\partial \Phi / \partial x_{i}$, then, in the limit $\epsilon \rightarrow 0$, the dSBKP equation reduces to

$$
\left[\ln \left(\frac{\Phi_{y}+\Phi_{t}}{\Phi_{y}-\Phi_{t}}\right)\right]_{x}=\left[\ln \left(\frac{\Phi_{t}+\Phi_{x}}{\Phi_{t}-\Phi_{x}}\right)\right]_{y}
$$

with $\left(x_{1}, x_{2}, x_{3}\right)=(x, y, t)$, while the linear system (5.6) becomes

$$
\Phi_{y}=\frac{c-1}{c+1} \Phi_{x}, \quad \Phi_{t}=\frac{a-1}{a+1} \Phi_{y}, \quad \Phi_{x}=\frac{b-1}{b+1} \Phi_{t} .
$$

The equation 7.5$)$ is invariant under transformations of the form $\Phi \rightarrow F(\Phi)$, where $F$ is an arbitrary differentiable function, and complex conjugation. Its solutions constitute mappings $\Phi: \mathbb{R}^{3} \rightarrow \mathbb{C}$ to which we shall refer as SBKP mappings.

In order to investigate the nature of SBKP mappings, it is first noted that the system (7.6) gives rise to the consistency condition

$$
\frac{a-1}{a+1} \frac{b-1}{b+1} \frac{c-1}{c+1}=1 .
$$

Accordingly, if we set $\gamma=(c-1) /(c+1)$ and $\delta=(b+1) /(b-1)$ then SBKP mappings are obtained by integrating the linear pair

$$
\Phi_{y}=\gamma \Phi_{x}, \quad \Phi_{t}=\delta \Phi_{x},
$$

where $\gamma, \delta$ are solutions of the coupled nonlinear system

$$
\gamma_{t}+\gamma \delta_{x}=\delta_{y}+\delta \gamma_{x}, \quad\left(1-\delta^{2}\right) \gamma_{t}=\left(1-\gamma^{2}\right) \delta_{y}
$$




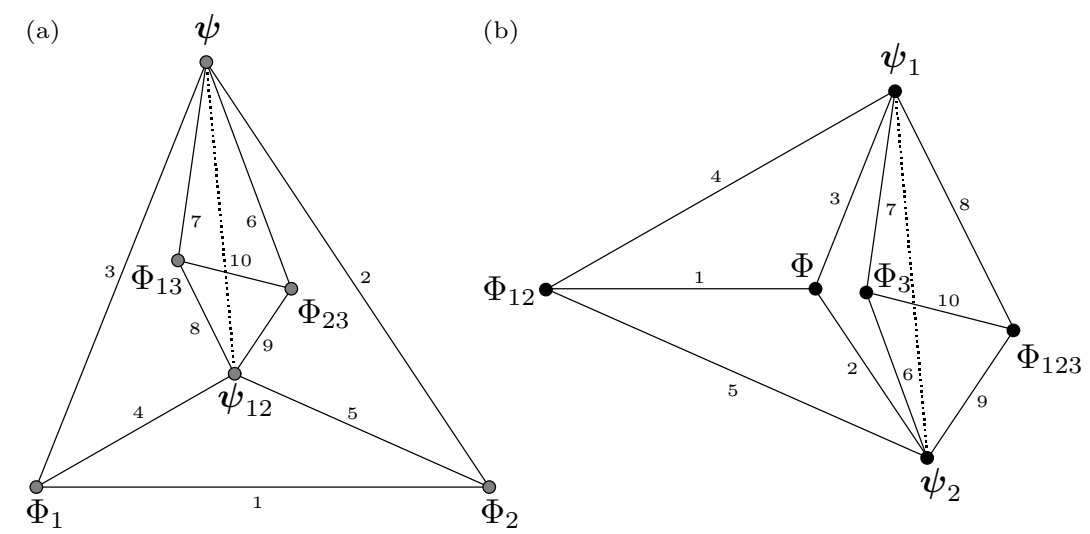

Figure 10: Reciprocal $(6,10)$ figures

The latter system comprises (7.5) and the compatibility condition for (7.6). In terms of the variables

$$
\mu=\frac{\mathrm{i}-\gamma}{\mathrm{i}+\gamma}, \quad z=x+\mathrm{i} y
$$

the linear system (7.8) reads

$$
\Phi_{\bar{z}}=\mu \Phi_{z}, \quad \Phi_{t}=\delta\left(\Phi_{z}+\Phi_{\bar{z}}\right) .
$$

Thus, SBKP mappings are descriptive of particular evolutions of quasi-conformal mappings if the variable $t$ is interpreted as time and the complex dilation $\mu$ in the Beltrami equation $(7.11)_{1}$ is assumed to be bounded [51]-[53]. In other words, SBKP mappings consist of one-parameter families of quasi-conformal mappings $\Phi(t): \mathbb{C} \rightarrow \mathbb{C}$. The solutions of the dSBKP equation therefore constitute integrable discretizations of particular families of quasi-conformal mappings.

\section{8 'Integrable' reciprocal $(6,10)$ figures, configu- rations and associated lattices}

It turns out that the dBKP equation is but the simplest integrable equation which may be derived from reciprocal figures. Another example is obtained if we remove two edges from Maxwell's 'octahedral' figure as displayed in Figure $3(\mathrm{a})$ in order to achieve determinacy. Indeed, Figure 10(a) now consists of six vertices, six polygons and ten edges and thus admits a reciprocal figure with the same number of vertices and edges which is uniquely determined up to an arbitrary scaling. We shall refer to reciprocal figures of this kind as reciprocal $(6,10)$ figures. 


\subsection{Reciprocal $(6,10)$ figures and configurations}

We first exploit the fact that Maxwell's theorem guarantees the existence of reciprocal $(6,10)$ figures and label the vertices by $\boldsymbol{\psi}, \boldsymbol{\psi}_{12}, \Phi_{1}, \Phi_{2}, \Phi_{13}, \Phi_{23}$ and $\boldsymbol{\psi}_{1}, \boldsymbol{\psi}_{2}, \Phi, \Phi_{3}, \Phi_{12}, \Phi_{123}$ respectively. Here, this particular labelling is of no relevance but will turn out to be of importance in connection with $(6,10)$ lattices. It proves convenient to group the conditions of parallelism into two sets of linear equations, namely

$$
\begin{array}{ll}
\Phi_{12}-\Phi=a\left(\Phi_{1}-\Phi_{2}\right), & \boldsymbol{\psi}_{1}-\Phi=d\left(\Phi_{1}-\boldsymbol{\psi}\right) \\
\Phi_{13}-\boldsymbol{\psi}=b\left(\boldsymbol{\psi}_{1}-\Phi_{3}\right), & \boldsymbol{\psi}_{2}-\Phi=e\left(\Phi_{2}-\boldsymbol{\psi}\right) \\
\Phi_{23}-\boldsymbol{\psi}=c\left(\boldsymbol{\psi}_{2}-\Phi_{3}\right) &
\end{array}
$$

and

$$
\begin{array}{ll}
\Phi_{123}-\Phi_{3}=a_{3}\left(\Phi_{13}-\Phi_{23}\right), & \boldsymbol{\psi}_{12}-\Phi_{2}=d_{2}\left(\Phi_{12}-\boldsymbol{\psi}_{2}\right) \\
\Phi_{123}-\boldsymbol{\psi}_{2}=b_{2}\left(\boldsymbol{\psi}_{12}-\Phi_{23}\right), & \boldsymbol{\psi}_{12}-\Phi_{1}=e_{1}\left(\Phi_{12}-\boldsymbol{\psi}_{1}\right) \\
\Phi_{123}-\boldsymbol{\psi}_{1}=c_{1}\left(\boldsymbol{\psi}_{12}-\Phi_{13}\right) &
\end{array}
$$

with associated real dilation coefficients $a, b, c, d, e$ and $a_{3}, b_{2}, c_{1}, d_{2}, e_{1}$. Elimination of $\boldsymbol{\psi}_{12}$ between $(8.2)_{2,4}$ on the one hand and $\boldsymbol{\psi}_{12}, \Phi_{123}$ between 8.2$)_{1,3,5}$ on the other hand results in

$$
\begin{aligned}
{\left[d_{2} a+e_{1}(d-a)-1\right]\left(\Phi_{1}-\boldsymbol{\psi}\right)+\left[e_{1} a-d_{2}(e+a)+1\right]\left(\Phi_{2}-\boldsymbol{\psi}\right) } & =0 \\
{\left[\left(c_{1} a_{3}-b_{2} c_{1}-a_{3} b_{2}\right) b\right.} & \left.+b_{2}\right]\left(\boldsymbol{\psi}_{1}-\Phi_{3}\right) \\
+\left[\left(b_{2} a_{3}-a_{3} c_{1}+c_{1} b_{2}\right) c-c_{1}\right]\left(\boldsymbol{\psi}_{2}-\Phi_{3}\right) & =0 .
\end{aligned}
$$

Since we consider the generic case, neither the vertices $\psi, \Phi_{1}, \Phi_{2}$ nor the vertices $\boldsymbol{\psi}_{1}, \boldsymbol{\psi}_{2}, \Phi_{3}$ are collinear. Hence, the relations (8.3) may be solved for $b_{2}, c_{1}, d_{2}, e_{1}$. The remaining consistency condition $\left.(8.2)_{3}-8.2\right)_{5}$ then reduces to

$$
\left\{a_{3}[b c(a d+e d-e a)+a(b-c)]-a\right\}\left(\boldsymbol{\psi}_{1}-\boldsymbol{\psi}_{2}\right)=0
$$

so that $a_{3}$ is likewise determined.

An algebraic proof of the existence of reciprocal $(6,10)$ figures is now readily obtained. Thus, given a generic $(6,10)$ figure with vertices $\boldsymbol{\psi}, \boldsymbol{\psi}_{12}, \Phi_{1}, \Phi_{2}$, $\Phi_{13}, \Phi_{23}$, we draw a line parallel to the edge $\left(\Phi_{1}, \Phi_{2}\right)$ and choose two points $\Phi$ and $\Phi_{12}$ on this line. The vertices $\boldsymbol{\psi}_{1}$ and $\boldsymbol{\psi}_{2}$ are then given by the points of intersection of the pairs of lines which pass through $\Phi$ and $\Phi_{12}$ and are parallel to the corresponding edges of the original figure (cf. Figure 10). The vertex $\Phi_{3}$ is constructed in a similar manner. In algebraic terms, this implies that the linear equations (8.1), 8.2 $)_{2,4}$ are satisfied with some real dilations $a, b, c, d, e$ and $d_{2}, e_{1}$. The latter two dilations are determined by $(8.3)_{1}$. Finally, the three lines which pass through $\boldsymbol{\psi}_{1}, \boldsymbol{\psi}_{2}, \Phi_{3}$ and are parallel to the edges $\left(\boldsymbol{\psi}_{12}, \Phi_{13}\right)$, $\left(\psi_{12}, \Phi_{23}\right),\left(\Phi_{13}, \Phi_{23}\right)$ respectively are concurrent since the remaining linear equations $(8.2)_{1,3,5}$ are compatible if the coefficients $a_{3}, b_{2}, c_{1}$ are defined by the compatibility conditions 8.3$)_{2}$ and $(8.4)$. Accordingly, the reciprocal $(6,10)$ 
figure exists and is defined up to an arbitrary scaling. An alternative method of constructing reciprocal $(6,10)$ figures is stated in the following theorem:

Theorem 5 (Reciprocal $(6,10)$ figures). The real dilations $a, b, c, d, e$ and $a_{3}, b_{2}, c_{1}, d_{2}, e_{1}$ as defined by (8.1) and (8.7) associated with two reciprocal $(6,10)$ configurations are related by

$$
\begin{gathered}
a_{3}=\frac{a}{b c(a d+e d-e a)+a(b-c)} \\
b_{2}=\frac{a_{3}(c-b)+1}{c}, \quad c_{1}=\frac{a_{3}(c-b)+1}{b} \\
d_{2}=\frac{d}{a d+e d-e a}, \quad e_{1}=\frac{e}{a d+e d-e a} .
\end{gathered}
$$

Conversely, let $\Phi, \Phi_{1}, \Phi_{2}, \Phi_{3}, \psi$ be five generic points on the complex plane, $a, b, c, d, e \in \mathbb{R}$ be five arbitrary non-vanishing real numbers and $a_{3}, b_{2}, c_{1}, d_{2}, e_{1}$ be given by (8.5). Then, the linear system (8.1), (8.9) is compatible and the points $\boldsymbol{\psi}, \boldsymbol{\psi}_{12}, \Phi_{1}, \Phi_{2}, \Phi_{13}, \Phi_{23}$ and $\boldsymbol{\psi}_{1}, \boldsymbol{\psi}_{2}, \Phi, \Phi_{3}, \Phi_{12}, \Phi_{123}$ constitute the vertices of two reciprocal $(6,10)$ figures. These obey the cross-ratio relations

$$
\begin{aligned}
Q\left(\Phi_{1}, \Phi_{2}, \boldsymbol{\psi}, \boldsymbol{\psi}_{12}\right) & =Q\left(\boldsymbol{\psi}_{2}, \boldsymbol{\psi}_{1}, \Phi_{12}, \Phi\right) \\
Q\left(\boldsymbol{\psi}_{1}, \boldsymbol{\psi}_{2}, \Phi_{3}, \Phi_{123}\right) & =Q\left(\Phi_{23}, \Phi_{13}, \boldsymbol{\psi}_{12}, \boldsymbol{\psi}\right) .
\end{aligned}
$$

The above cross-ratio relations are a consequence of the identities $c b_{2}=b c_{1}$ and $e d_{2}=d e_{1}$. They express the fact that the quadruplets $\left(\Phi_{1}, \Phi_{2}, \boldsymbol{\psi}, \boldsymbol{\psi}_{12}\right)$ and $\left(\boldsymbol{\psi}_{2}, \boldsymbol{\psi}_{1}, \Phi_{12}, \Phi\right)$ on the one hand and $\left(\Phi_{23}, \Phi_{13}, \boldsymbol{\psi}_{12}, \boldsymbol{\psi}\right)$ and $\left(\boldsymbol{\psi}_{1}, \boldsymbol{\psi}_{2}, \Phi_{3}, \Phi_{123}\right)$ on the other hand give rise to two pairs of reciprocal triangles if the parallel line segments $\left(\boldsymbol{\psi}, \boldsymbol{\psi}_{12}\right)$ and $\left(\boldsymbol{\psi}_{1}, \boldsymbol{\psi}_{2}\right)$ are drawn (cf. Figure 10). The occurrence of cross-ratios once again suggests that reciprocal $(6,10)$ figures should be regarded as degenerate reciprocal $(6,10)$ configurations. Indeed, if, in a $(6,10)$ figure, we replace the straight edges by circular arcs whose extensions meet at a point then two such $(6,10)$ configurations are reciprocally related if corresponding angles are equal. As in the case of reciprocal $(4,6)$ configurations, it is now straight forward to show that reciprocal $(6,10)$ configurations are governed by the crossratio relations (8.6) and the associated complex dilations satisfy the algebraic relations (8.5).

\subsection{Reciprocal $(6,10)$ lattices}

As in the case of reciprocal triangles, it is now possible to define lattices which consist of reciprocal $(6,10)$ figures and configurations. Thus, we consider two three-dimensional lattices on the complex plane of the same combinatorics and label them as follows. The sets $G^{(0)}$ and $G^{(1)}$ are defined as in Section 5, that is

$$
\begin{aligned}
& G^{(0)}=\left\{\left(n_{1}, n_{2}, n_{4}\right) \in \mathbb{Z}^{3}: n_{1}+n_{2}+n_{4} \text { even }\right\} \\
& G^{(1)}=\left\{\left(n_{1}, n_{2}, n_{4}\right) \in \mathbb{Z}^{3}: n_{1}+n_{2}+n_{4} \text { odd }\right\} .
\end{aligned}
$$



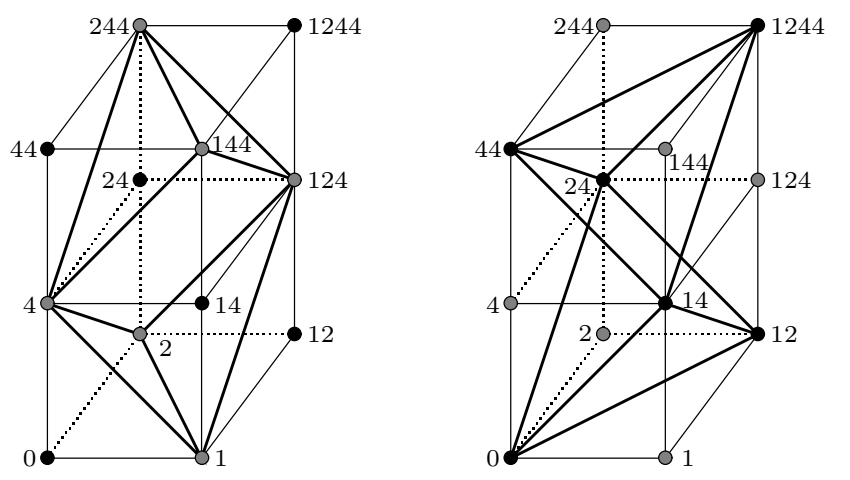

Figure 11: The elementary cells of $G^{(1)}$ and $G^{(0)}$

However, the edge structure of the lattices associated with $G^{(0)}$ and $G^{(1)}$ is slightly modified in order to take into account that the reciprocal figures displayed in Figure 10 consist of two pairs of reciprocal triangles if the dotted lines are included. Accordingly, every second 'horizontal' edge which joins two tetrahedra placed on top of each other is removed from the lattices associated with $G^{(0)}$ and $G^{(1)}$. This is indicated in Figure 11. Hence, the polyhedron inscribed in eight cubes of the $\mathbb{Z}^{3}$ lattice constitutes a stella octangula without the central horizontal edges (cf. Figure 9). Due to the complementary character of $G^{(0)}$ and $G^{(1)}$, the associated lattices $\Phi^{(0)}$ and $\Phi^{(1)}$ may now be combined to a lattice

$$
\hat{\Phi}(\boldsymbol{n})=\Phi^{(i)}(\boldsymbol{n}) \text { if } \boldsymbol{n} \in G^{(i)}, \quad \boldsymbol{n}=\left(n_{1}, n_{2}, n_{4}\right) .
$$

In order to simplify the book-keeping which takes care of the missing horizontal edges, it is convenient to introduce two lattices $\Phi$ and $\boldsymbol{\psi}$ according to

$$
\Phi\left(n_{1}, n_{2}, n_{3}\right)=\hat{\Phi}\left(n_{1}, n_{2}, 2 n_{3}\right), \quad \boldsymbol{\psi}\left(n_{1}, n_{2}, n_{3}\right)=\hat{\Phi}\left(n_{1}, n_{2}, 2 n_{3}+1\right) .
$$

As a consequence of Theorem 5, the consistency conditions (8.3) and (8.4) may be interpreted as the compatibility conditions for the linear difference equations (8.1) which guarantee the existence of two three-dimensional lattices $\Phi$ and $\boldsymbol{\psi}$ on the complex plane which encapsulate an infinite number of reciprocal $(6,10)$ figures or configurations via their constituent sublattices $\Phi^{(0)}$ and $\Phi^{(1)}$. By virtue of the identities $c b_{2}=b c_{1}, e d_{2}=d e_{1}$ and $c d a_{3}=a c_{1} d_{2}$, the dilations may be parametrized in terms of two functions $\tau$ and $\sigma$. The relations (8.5), now regarded as difference equations, then reduce to a system of two integrable equations. This is the content of the following theorem:

Theorem $6((6,10)$ lattices $)$. Two three-dimensional lattices $\Phi$ and $\boldsymbol{\psi}$ or, equivalently, $\Phi^{(0)}$ and $\Phi^{(1)}$ on the complex plane consist of reciprocal $(6,10)$ configurations (figures) with vertices $\boldsymbol{\psi}, \boldsymbol{\psi}_{12}, \Phi_{1}, \Phi_{2}, \Phi_{13}, \Phi_{23}$ and $\boldsymbol{\psi}_{1}, \boldsymbol{\psi}_{2}, \Phi, \Phi_{3}$, 
$\Phi_{12}, \Phi_{123}$ if and only if the complex (real) dilations defined by

$$
\begin{array}{rlrl}
\Phi_{12}-\Phi & =\frac{\tau_{1} \tau_{2}}{\tau \tau_{12}}\left(\Phi_{1}-\Phi_{2}\right), & \boldsymbol{\psi}_{1}-\Phi=\frac{\tau_{1} \sigma}{\tau \sigma_{1}}\left(\Phi_{1}-\boldsymbol{\psi}\right) \\
\Phi_{13}-\boldsymbol{\psi}=\frac{\sigma_{1} \tau_{3}}{\sigma \tau_{13}}\left(\boldsymbol{\psi}_{1}-\Phi_{3}\right), & \boldsymbol{\psi}_{2}-\Phi=\frac{\tau_{2} \sigma}{\tau \sigma_{2}}\left(\Phi_{2}-\boldsymbol{\psi}\right) \\
\Phi_{23}-\boldsymbol{\psi}=\frac{\sigma_{2} \tau_{3}}{\sigma \tau_{23}}\left(\boldsymbol{\psi}_{2}-\Phi_{3}\right) & &
\end{array}
$$

may be parametrized in terms of solutions of the coupled BKP-type system

$$
\begin{aligned}
\sigma_{12} \tau+\sigma_{1} \tau_{2} & =\sigma \tau_{12}+\sigma_{2} \tau_{1} \\
\sigma \tau_{123}+\sigma_{2} \tau_{13} & =\sigma_{12} \tau_{3}+\sigma_{1} \tau_{23} .
\end{aligned}
$$

Lattices of this type are equivalently described by the cross-ratio relations

$$
\begin{aligned}
Q\left(\Phi_{1}, \Phi_{2}, \boldsymbol{\psi}, \boldsymbol{\psi}_{12}\right) & =Q\left(\boldsymbol{\psi}_{2}, \boldsymbol{\psi}_{1}, \Phi_{12}, \Phi\right) \\
Q\left(\boldsymbol{\psi}_{1}, \boldsymbol{\psi}_{2}, \Phi_{3}, \Phi_{123}\right) & =Q\left(\Phi_{23}, \Phi_{13}, \boldsymbol{\psi}_{12}, \boldsymbol{\psi}\right) .
\end{aligned}
$$

It turns out that the above results may be generalized. It is indeed possible to define integrable systems on three-dimensional hybrids of octahedral and hexahedral lattices which give rise to lattices on the complex plane composed of Maxwell's complete octahedral figures and their hexahedral reciprocals. This is briefly discussed in the final section.

\section{Reciprocal octahedral and hexahedral figures}

We begin with an observation associated with reciprocal triangles and $(6,10)$ figures. Thus, the 8-point relation (3.6) may be interpreted as the equality of the cross-ratios associated with any two 'parallel' quadrilaterals which reside in reciprocal triangles. Indeed, for instance, the closed polygon

$$
\Phi_{1} \rightarrow \Phi_{2} \rightarrow \Phi_{3} \rightarrow \Phi_{123} \rightarrow \Phi_{1}
$$

in Figure 4 admits the counterpart

$$
\Phi_{12} \rightarrow \Phi \rightarrow \Phi_{23} \rightarrow \Phi_{13} \rightarrow \Phi_{12}
$$

with corresponding parallel edges and the 8-point relation may be written as

$$
Q\left(\Phi_{1}, \Phi_{2}, \Phi_{3}, \Phi_{123}\right)=Q\left(\Phi_{12}, \Phi, \Phi_{23}, \Phi_{13}\right) .
$$

The cross-ratio relations (8.6) associated with the reciprocal $(6,10)$ figures depicted in Figure 10 admit the same interpretation. Moreover, these imply that, for instance,

$$
M\left(\Phi_{1}, \Phi_{2}, \boldsymbol{\psi}, \Phi_{23}, \Phi_{13}, \boldsymbol{\psi}_{12}\right)=M\left(\Phi_{12}, \Phi, \boldsymbol{\psi}_{2}, \Phi_{3}, \Phi_{123}, \boldsymbol{\psi}_{1}\right)
$$




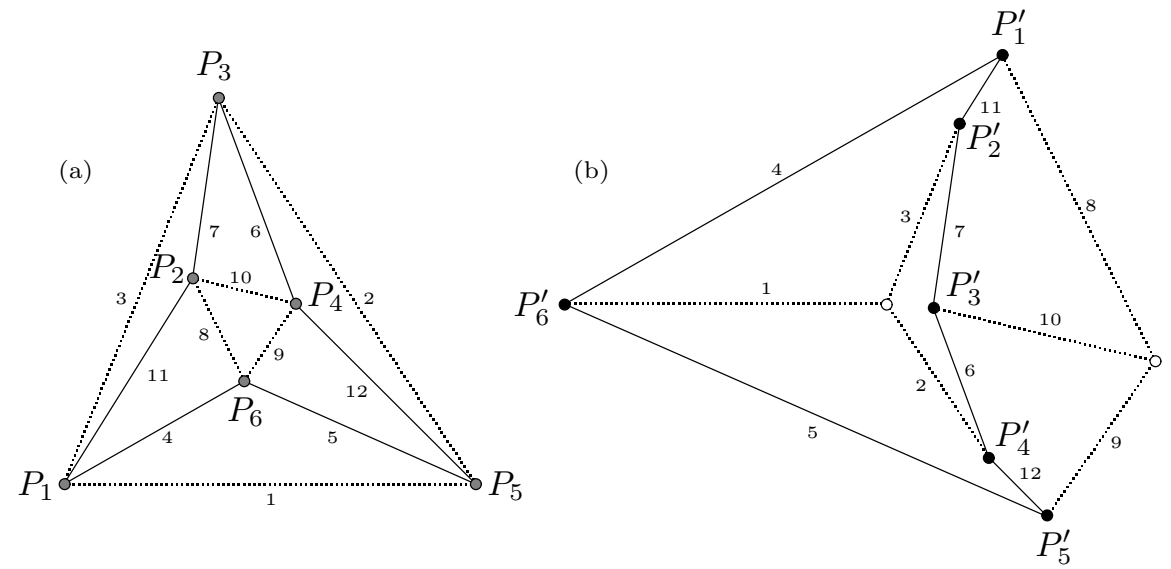

Figure 12: 'Parallel' hexagons $H$

where the multi-ratio of six points $P_{1}, \ldots, P_{6}$ on the complex plane is defined by

$$
M\left(P_{1}, P_{2}, P_{3}, P_{4}, P_{5}, P_{6}\right)=\frac{\left(P_{1}-P_{2}\right)\left(P_{3}-P_{4}\right)\left(P_{5}-P_{6}\right)}{\left(P_{2}-P_{3}\right)\left(P_{4}-P_{5}\right)\left(P_{6}-P_{1}\right)} .
$$

Inspection of Figure 10 reveals that the points $\Phi_{1}, \Phi_{2}, \boldsymbol{\psi}, \Phi_{23}, \Phi_{13}, \boldsymbol{\psi}_{12}$ and $\Phi_{12}, \Phi, \boldsymbol{\psi}_{2}, \Phi_{3}, \Phi_{123}, \boldsymbol{\psi}_{1}$ constitute the vertices of two 'parallel' hexagons which are generated by moving along the edges $1,2,6,10,8$ and 4 . We observe in passing that, up to complex conjugation, the multi-ratio is invariant under the group of inversive transformations. This has been exploited in [28] to relate the discrete Schwarzian KP equation to the fundamental Theorem of Menelaus in the setting of plane inversive geometry.

We now focus on Maxwell's octahedral figure and its hexahedral reciprocals as displayed in Figure 3. It is readily seen that there exist four pairs of parallel hexagons. If the ordered collections of points $P_{1}, \ldots, P_{6}$ and $P_{1}^{\prime}, \ldots, P_{6}^{\prime}$ denote the vertices of a hexagon

$$
H\left(P_{1}, P_{2}, P_{3}, P_{4}, P_{5}, P_{6}\right)
$$

and its parallel companion

$$
H\left(P_{1}^{\prime}, P_{2}^{\prime}, P_{3}^{\prime}, P_{4}^{\prime}, P_{5}^{\prime}, P_{6}^{\prime}\right)
$$

respectively as shown in Figure 12 then real dilations $\alpha_{i k}$ may be introduced according to

$$
P_{i}^{\prime}-P_{k}^{\prime}=\alpha_{i k}\left(P_{i}-P_{k}\right) .
$$

Another figure which turns out to be significant is that of two triangles joined by a vertex. Maxwell's octahedral figure contains twelve objects of this kind, an example of which together with the associated parallel figure in the hexahedral reciprocal is shown in Figure 13. The edges of the pair of triangles

$$
T\left(\boldsymbol{v}_{1}, \boldsymbol{v}_{2}, \boldsymbol{v}_{3}, \boldsymbol{v}_{4}, \boldsymbol{v}_{5}, \boldsymbol{v}_{6}\right)
$$



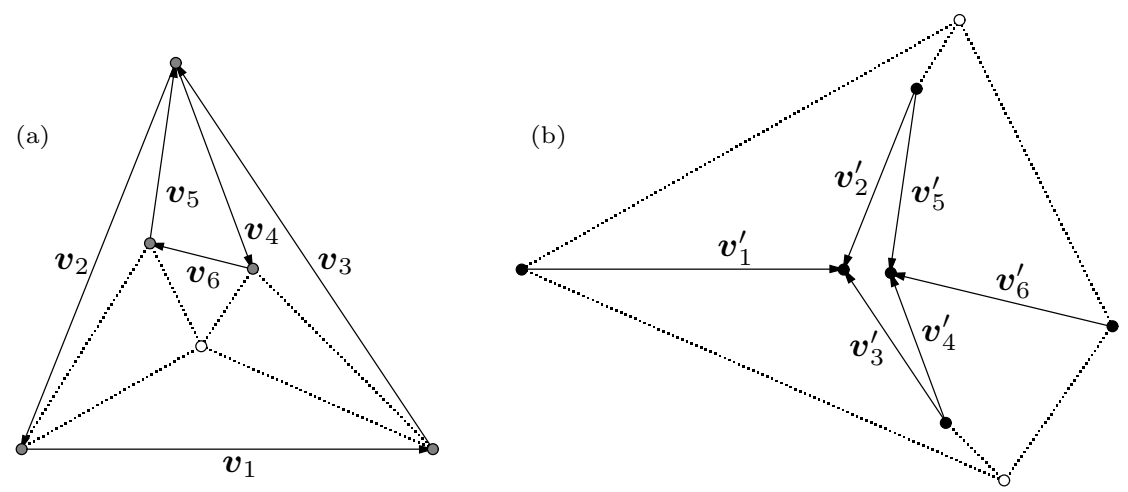

Figure 13: 'Parallel' figures $T$ and $T^{\prime}$

are denoted by $\boldsymbol{v}_{1}, \ldots, \boldsymbol{v}_{6}$ while the edges of the parallel figure

$$
T^{\prime}\left(\boldsymbol{v}_{1}^{\prime}, \boldsymbol{v}_{2}^{\prime}, \boldsymbol{v}_{3}^{\prime}, \boldsymbol{v}_{4}^{\prime}, \boldsymbol{v}_{5}^{\prime}, \boldsymbol{v}_{6}^{\prime}\right)
$$

are labelled by $\boldsymbol{v}_{1}^{\prime}, \ldots, \boldsymbol{v}_{6}^{\prime}$. The orientation of the vectors $\boldsymbol{v}_{i}$ and $\boldsymbol{v}_{i}^{\prime}$ is prescribed as follows. The vectors $\boldsymbol{v}_{i}^{\prime}$ point towards the two vertices of $T^{\prime}$ which are dual to the two triangles in $T$, that is the vertices which are enclosed by $\boldsymbol{v}_{1}^{\prime}, \boldsymbol{v}_{2}^{\prime}, \boldsymbol{v}_{3}^{\prime}$ and $\boldsymbol{v}_{4}^{\prime}, \boldsymbol{v}_{5}^{\prime}, \boldsymbol{v}_{6}^{\prime}$ respectively. We assign a counterclockwise (or clockwise) orientation to the quadrilateral in $T^{\prime}$, that is we regard the quadrilateral as being generated by moving successively along the edges associated with $\boldsymbol{v}_{2}^{\prime}, \boldsymbol{v}_{3}^{\prime}, \boldsymbol{v}_{4}^{\prime}, \boldsymbol{v}_{5}^{\prime}$, say. The orientation of the degenerate hexagon $T$ is defined to be that of the quadrilateral in the sense that we follow the edges in the same order, that is $\boldsymbol{v}_{2},\left(\boldsymbol{v}_{1}\right), \boldsymbol{v}_{3}, \boldsymbol{v}_{4},\left(\boldsymbol{v}_{6}\right), \boldsymbol{v}_{5}$. This determines the direction of the vectors $\boldsymbol{v}_{i}$. Associated dilations $\alpha_{i}$ are then given by

$$
\boldsymbol{v}_{i}^{\prime}=\alpha_{i} \boldsymbol{v}_{i} .
$$

In Section 2, it has been demonstrated that any octahedral figure is associated with a two-parameter family of hexahedral reciprocals. As usual, we here regard figures which are similar as identical. This may now be exploited to construct a one-parameter family of reciprocals which has distinct properties. This is encapsulated in the following theorem which is a consequence of the analysis conducted below.

Theorem 7 (A canonical class of reciprocal octahedral and hexahedral figures.) Any octahedral figure admits a one-parameter family of hexahedral reciprocals such that any two parallel hexagons $H\left(P_{1}, P_{2}, P_{3}, P_{4}, P_{5}, P_{6}\right)$ and $H\left(P_{1}^{\prime}, P_{2}^{\prime}, P_{3}^{\prime}, P_{4}^{\prime}, P_{5}^{\prime}, P_{6}^{\prime}\right)$ and any two parallel figures $T\left(\boldsymbol{v}_{1}, \boldsymbol{v}_{2}, \boldsymbol{v}_{3}, \boldsymbol{v}_{4}, \boldsymbol{v}_{5}, \boldsymbol{v}_{6}\right)$ 


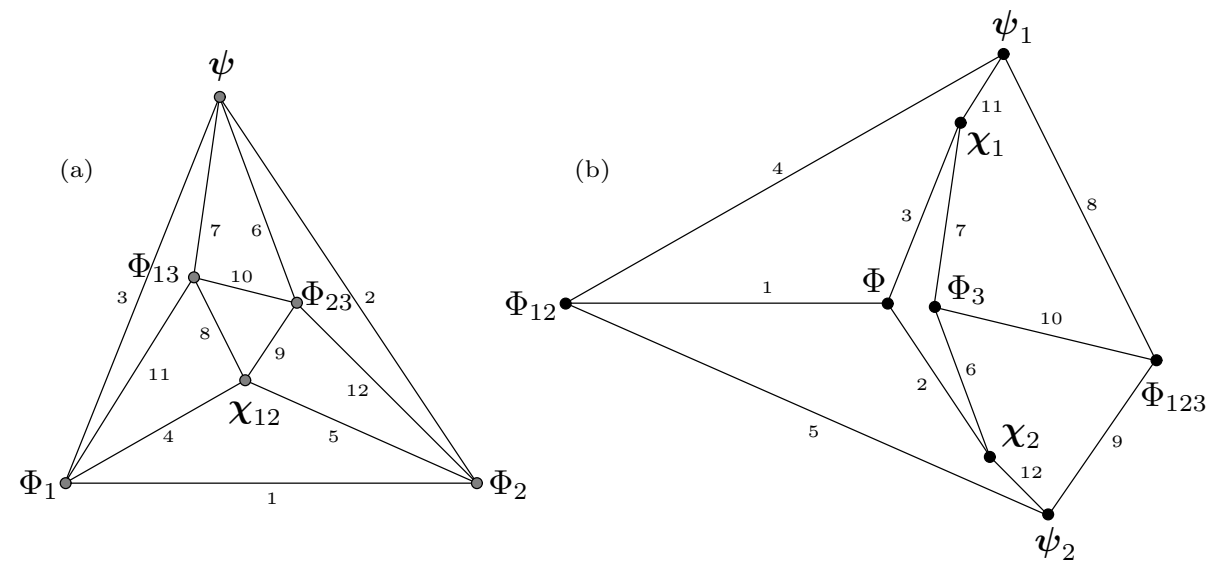

Figure 14: A parametrization of reciprocal octahedral and hexahedral figures

and $T^{\prime}\left(\boldsymbol{v}_{1}^{\prime}, \boldsymbol{v}_{2}^{\prime}, \boldsymbol{v}_{3}^{\prime}, \boldsymbol{v}_{4}^{\prime}, \boldsymbol{v}_{5}^{\prime}, \boldsymbol{v}_{6}^{\prime}\right)$ as defined above give rise to the algebraic identities

$$
\begin{gathered}
\frac{\alpha_{12} \alpha_{34} \alpha_{56}}{\alpha_{23} \alpha_{45} \alpha_{61}}=1 \\
\alpha_{2}+\alpha_{3}+\frac{\alpha_{2} \alpha_{3}}{\alpha_{1}}+\alpha_{4}+\alpha_{5}+\frac{\alpha_{4} \alpha_{5}}{\alpha_{6}}=0 \\
M\left(P_{1}, P_{2}, P_{3}, P_{4}, P_{5}, P_{6}\right)=M\left(P_{1}^{\prime}, P_{2}^{\prime}, P_{3}^{\prime}, P_{4}^{\prime}, P_{5}^{\prime}, P_{6}^{\prime}\right) .
\end{gathered}
$$

Even though there exist four pairs of parallel hexagons and twelve pairs of parallel figures $T$ and $T^{\prime}$, only six of the identities $(9.12)_{1,2}$ are independent. Moreover, the relations (9.12) $)_{1,3}$ are identical by virtue of the definitions (9.8). In the context of octahedral and hexahedral integrable lattices (cf. Section 9.2), these may be satisfied identically by introducing 'tau-functions'. The remaining (three) relations $(9.12)_{2}$ are interpreted once again as a discrete integrable system.

\subsection{An algebraic characterization}

The octahedral figure 3(a) may be obtained from the $(6,10)$ figure 10 (a) by adding the edges 11 and 12 . From a combinatorial point of view, this amounts to replacing each of the vertices $\boldsymbol{\psi}_{1}$ and $\boldsymbol{\psi}_{2}$ in the reciprocal figure 10 (b) by an edge and two vertices. Accordingly, it is natural to adopt the parametrization shown in Figure 14. Indeed, if the vertices $\boldsymbol{\chi}_{1}, \boldsymbol{\chi}_{2}$ and $\boldsymbol{\psi}_{1}, \boldsymbol{\psi}_{2}$ coincide then Figure 14(b) reduces to Figure 10(b). The conditions of parallelism are now naturally split into the two groups of linear equations

$$
\begin{array}{ll}
\Phi_{12}-\Phi=a\left(\Phi_{1}-\Phi_{2}\right), & \chi_{1}-\Phi=d\left(\Phi_{1}-\boldsymbol{\psi}\right) \\
\Phi_{13}-\boldsymbol{\psi}=b\left(\chi_{1}-\Phi_{3}\right), & \chi_{2}-\Phi=e\left(\Phi_{2}-\boldsymbol{\psi}\right) \\
\Phi_{23}-\boldsymbol{\psi}=c\left(\boldsymbol{\chi}_{2}-\Phi_{3}\right) &
\end{array}
$$


and

$$
\begin{array}{ll}
\Phi_{123}-\Phi_{3}=a_{3}\left(\Phi_{13}-\Phi_{23}\right), & \chi_{12}-\Phi_{2}=d_{2}\left(\Phi_{12}-\boldsymbol{\psi}_{2}\right) \\
\Phi_{123}-\boldsymbol{\psi}_{2}=b_{2}\left(\chi_{12}-\Phi_{23}\right), & \chi_{12}-\Phi_{1}=e_{1}\left(\Phi_{12}-\boldsymbol{\psi}_{1}\right) \\
\Phi_{123}-\boldsymbol{\psi}_{1}=c_{1}\left(\boldsymbol{\chi}_{12}-\Phi_{13}\right) &
\end{array}
$$

and the pair

$$
\begin{aligned}
& \boldsymbol{\psi}_{1}-\boldsymbol{\chi}_{1}=g\left(\Phi_{13}-\Phi_{1}\right) \\
& \boldsymbol{\psi}_{2}-\boldsymbol{\chi}_{2}=h\left(\Phi_{23}-\Phi_{2}\right)
\end{aligned}
$$

with real dilations $a, b, c, d, e, a_{3}, b_{2}, c_{1}, d_{2}, e_{1}$ and $g, h$. Elimination of $\chi_{12}$ and $\Phi_{123}$ from (9.14) leads to three relations of the form

$$
E^{1 k}\left(\Phi_{1}-\boldsymbol{\psi}\right)+E^{2 k}\left(\Phi_{2}-\boldsymbol{\psi}\right)+E^{3 k}\left(\Phi_{3}-\Phi\right)=0, \quad k=1,2,3,
$$

where the real coefficients $E^{i k}$ are given in terms of the dilations. In contrast to the cases discussed in the preceding, these relations do not imply that the coefficients $E^{i k}$ must vanish. However, in view of a connection with discrete integrable systems, it is canonical to assume that

$$
E^{i k}=0 .
$$

It turns out that only six of the latter conditions on the dilations are independent. If we now regard the vertices $\Phi, \Phi_{1}, \Phi_{2}, \Phi_{3}$ and $\boldsymbol{\psi}$ as given then the system (9.13)-(9.15) is compatible modulo (9.16). Since we have five points and six dilations at our disposal, the class of reciprocal octahedral and hexahedral figures constructed in this manner contains sixteen arbitrary real parameters. On the other hand, it is not difficult to show that any octahedral figure is represented by this class. Accordingly, if we specify an octahedral figure and the point $\Phi$, say, then there exist two degrees of freedom in the construction of the hexahedral reciprocal. Thus, if we neglect the usual arbitrary scaling then the following theorem obtains:

Theorem 8 (Construction of one-parameter families of reciprocal octahedral and hexahedral figures.) Any octahedral figure admits a oneparameter family of hexahedral reciprocals with $E^{i k}=0$, that is

$$
\begin{aligned}
a_{3} b d=b_{2} d_{2} a, \quad a_{3} c e & =c_{1} e_{1} a, \quad b_{2} e g=c_{1} d h \\
\frac{1}{a_{3} b c}+\frac{1}{b}-\frac{1}{c} & =\frac{d e}{a}+d-e \\
\frac{a e_{1}}{d_{2}}+\frac{1}{d_{2}}-a & =c e h+e-h \\
\frac{d}{b g}-\frac{1}{b}+d & =\frac{e}{c h}-\frac{1}{c}+e
\end{aligned}
$$


where the real dilations $a, \ldots, h$ are defined by (9.13)-(9.19). The vertices of these figures obey the multi-ratio relations

$$
\begin{aligned}
& M\left(\Phi_{23}, \Phi_{13}, \boldsymbol{\psi}, \Phi_{1}, \Phi_{2}, \boldsymbol{\chi}_{12}\right)=M\left(\Phi_{123}, \Phi_{3}, \boldsymbol{\chi}_{1}, \Phi, \Phi_{12}, \boldsymbol{\psi}_{2}\right) \\
& M\left(\Phi_{13}, \Phi_{23}, \boldsymbol{\psi}, \Phi_{2}, \Phi_{1}, \boldsymbol{\chi}_{12}\right)=M\left(\Phi_{123}, \Phi_{3}, \boldsymbol{\chi}_{2}, \Phi, \Phi_{12}, \boldsymbol{\psi}_{1}\right) \\
& M\left(\boldsymbol{\chi}_{12}, \Phi_{23}, \Phi_{2}, \boldsymbol{\psi}, \Phi_{1}, \Phi_{13}\right)=M\left(\Phi_{123}, \boldsymbol{\psi}_{2}, \boldsymbol{\chi}_{2}, \Phi, \boldsymbol{\chi}_{1}, \boldsymbol{\psi}_{1}\right) .
\end{aligned}
$$

Conversely, let $\Phi, \Phi_{1}, \Phi_{2}, \Phi_{3}, \boldsymbol{\psi}$ be five generic points on the complex plane, $a, b, c, d, e, g$ be six arbitrary non-vanishing real numbers and $a_{3}, b_{2}, c_{1}, d_{2}, e_{1}, h$ be given by (9.18). Then, the linear system (9.13)-(9.13) is compatible and the points $\boldsymbol{\psi}, \boldsymbol{\chi}_{12}, \Phi_{1}, \Phi_{2}, \Phi_{13}, \Phi_{23}$ and $\boldsymbol{\psi}_{1}, \boldsymbol{\psi}_{2}, \boldsymbol{\chi}_{1}, \boldsymbol{\chi}_{2}, \Phi, \Phi_{3}, \Phi_{12}, \Phi_{123}$ constitute the vertices of a reciprocal pair of octahedral and hexahedral figures.

The relations (9.18) are nothing but a set of six independent relations of the form $(9.12)_{1,2}$. Similarly, the multi-ratio relations (9.19) constitute three independent relations of the type $(9.12)_{3}$. In this connection, it is emphasized that for a given octahedral figure, the multi-ratio conditions $(9.12)_{3}$ define the one-parameter family of hexahedral reciprocals.

\subsection{Integrable lattices}

In the case of reciprocal triangles and $(6,10)$ figures, the reciprocals are of the same type as the original figures. Accordingly, the associated pairs of integrable lattices consist of two lattices of the same kind. In the present situation, the reciprocal figures exhibit different combinatorics. It would therefore be natural to seek two lattices which consist of octahedral and hexahedral figures respectively. Here, we adopt an alternative approach and consider two correlated lattices of the same kind, each of which contains both octahedral and hexahedral figures. Thus, we are concerned with lattices of the form

$$
\Phi^{(0)}: G^{(0)} \rightarrow \mathbb{C}, \quad \Phi^{(1)}: G^{(1)} \rightarrow \mathbb{C},
$$

where the set $G^{(0)}$ is given by

$$
\begin{aligned}
G^{(0)}=\left\{\left(n_{1}, n_{2}, n_{4}\right) \in \mathbb{Z}^{3}:\right. & n_{4}=0 \bmod 3 \text { if } n_{1}+n_{2} \text { even } \\
n_{4} & \left.=1,2 \bmod 3 \text { if } n_{1}+n_{2} \text { odd }\right\}
\end{aligned}
$$

and $G^{(1)}$ is the complement of $G^{(0)}$. The edge structure of $G^{(0)}$ and $G^{(1)}$ is obtained by alternating the octahedron and hexahedron displayed in Figure 15 in the two horizontal directions. In the vertical direction, polyhedra of the same type are stacked on top of each other. Thus, the lattices $\Phi^{(0)}$ and $\Phi^{(1)}$ possess the combinatorics of three-dimensional hybrids of octahedral and hexahedral lattices. In order to show that the relations (9.18) may be interpreted as a discrete integrable system defined on these lattices of non-trivial combinatorics, it is once again convenient to combine the (vertices of the) lattices $\Phi^{(i)}$ to one lattice

$$
\hat{\Phi}(\boldsymbol{n})=\Phi^{(i)}(\boldsymbol{n}) \text { if } \boldsymbol{n} \in G^{(i)}, \quad \boldsymbol{n}=\left(n_{1}, n_{2}, n_{4}\right)
$$



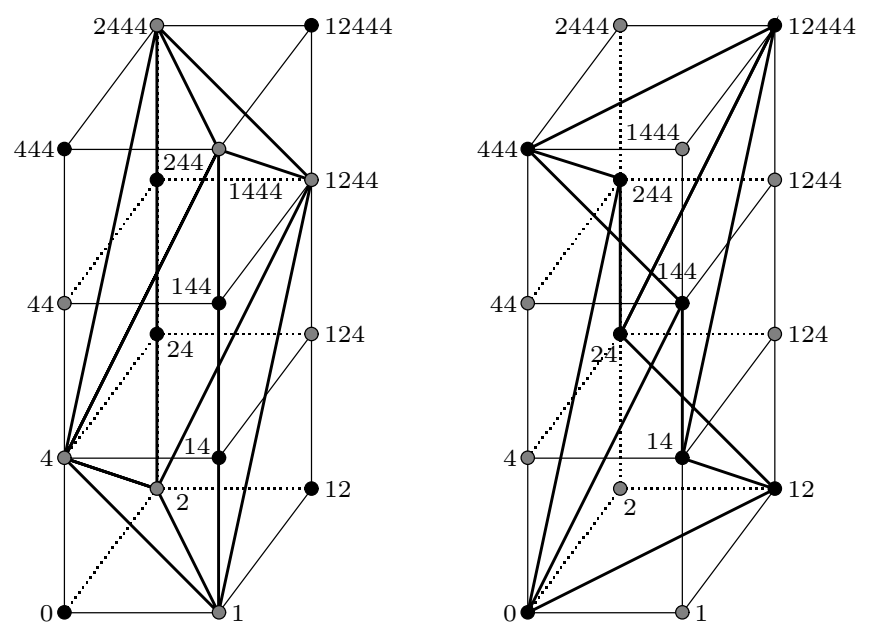

Figure 15: The building blocks of $G^{(0)}$ and $G^{(1)}$

and introduce the notation

$$
\begin{gathered}
\Phi\left(n_{1}, n_{2}, n_{3}\right)=\hat{\Phi}\left(n_{1}, n_{2}, 3 n_{3}\right), \quad \boldsymbol{\psi}\left(n_{1}, n_{2}, n_{3}\right)=\hat{\Phi}\left(n_{1}, n_{2}, 3 n_{3}+1\right) \\
\chi\left(n_{1}, n_{2}, n_{3}\right)=\hat{\Phi}\left(n_{1}, n_{2}, 3 n_{3}+2\right) .
\end{gathered}
$$

If we now regard indices on objects as shifts along corresponding edges then the algebraic system (9.13)-(9.15) may be regarded as a system of linear difference equations for the lattices $\Phi^{(0)}$ and $\Phi^{(1)}$ encapsulated in the fields $\Phi, \boldsymbol{\psi}$ and $\chi$. The compatibility conditions which guarantee the existence of $\Phi$ and $\chi$ are given by $(9.16)$ and are therefore satisfied if we assume that $E^{i k}=0$. The compatibility condition $\left(\boldsymbol{\psi}_{1}\right)_{2}=\left(\boldsymbol{\psi}_{2}\right)_{1}$ applied to the pair (9.15) results in the additional constraint

$$
g_{2}=h_{1} .
$$

The latter and the relations $(9.18)_{1,2,3}$, regarded as difference equations, give rise to a parametrization of the dilations in terms of 'tau-functions' $\tau, \kappa$ and $\nu$ according to

$$
\begin{gathered}
a=\frac{\tau_{1} \tau_{2}}{\tau \tau_{12}}, \quad b=\frac{\kappa_{1} \tau_{3}}{\nu \tau_{13}}, \quad c=\frac{\kappa_{2} \tau_{3}}{\nu \tau_{23}} \\
d=\frac{\nu \tau_{1}}{\kappa_{1} \tau}, \quad e=\frac{\nu \tau_{2}}{\kappa_{2} \tau}, \quad g=\frac{\tau_{1} \tau_{13}}{\kappa_{1} \nu_{1}}, \quad h=\frac{\tau_{2} \tau_{23}}{\kappa_{2} \nu_{2}} .
\end{gathered}
$$

The remaining relations 9.18$)_{4,5,6}$ then reduce to a 'trilinear' integrable system with (9.13), (9.15) being its linear representation. Thus, the following theorem holds: 
Theorem 9 (Integrable octahedral-hexahedral lattices.) The lattice equations

$$
\begin{aligned}
\Phi_{12}-\Phi & =\frac{\tau_{1} \tau_{2}}{\tau \tau_{12}}\left(\Phi_{1}-\Phi_{2}\right), & \chi_{1}-\Phi & =\frac{\nu \tau_{1}}{\kappa_{1} \tau}\left(\Phi_{1}-\psi\right) \\
\Phi_{13}-\psi & =\frac{\kappa_{1} \tau_{3}}{\nu \tau_{13}}\left(\chi_{1}-\Phi_{3}\right), & \chi_{2}-\Phi & =\frac{\nu \tau_{2}}{\kappa_{2} \tau}\left(\Phi_{2}-\psi\right) \\
\Phi_{23}-\psi & =\frac{\kappa_{2} \tau_{3}}{\nu \tau_{23}}\left(\chi_{2}-\Phi_{3}\right), & \psi-\chi & =\frac{\tau \tau_{3}}{\kappa \nu}\left(\Phi_{3}-\Phi\right)
\end{aligned}
$$

are compatible modulo the difference equations

$$
\begin{aligned}
\nu\left(\tau \tau_{123}-\tau_{3} \tau_{12}\right)+\kappa_{1}\left(\tau_{2} \tau_{3}-\tau \tau_{23}\right)+\kappa_{2}\left(\tau \tau_{13}-\tau_{1} \tau_{3}\right) & =0 \\
\kappa_{2}\left(\tau \kappa_{12}-\tau_{1} \nu_{2}\right)+\tau_{2}\left(\nu_{1} \kappa_{2}-\tau_{3} \tau_{12}\right)+\tau_{12}\left(\tau \tau_{23}-\nu \nu_{2}\right) & =0 \\
\nu\left(\kappa_{1} \nu_{2}-\kappa_{2} \nu_{1}\right)+\kappa_{1}\left(\tau_{2} \tau_{3}-\tau \tau_{23}\right)+\kappa_{2}\left(\tau \tau_{13}-\tau_{1} \tau_{3}\right) & =0 .
\end{aligned}
$$

The lattices $\Phi^{(0)}$ and $\Phi^{(1)}$ encapsulated in $\Phi, \boldsymbol{\psi}$ and $\boldsymbol{\chi}$ are composed of reciprocal octahedral and hexahedral figures obeying the multi-ratio relations 9.19).

We observe that the relations $(9.27)_{1,3}$ imply that

$$
\tau \tau_{123}-\tau_{3} \tau_{12}=\kappa_{1} \nu_{2}-\kappa_{2} \nu_{1} .
$$

There exists a variety of other reciprocal figures which may be associated with cross-ratio and multi-ratio relations and are therefore naturally placed in the setting of inversive geometry. Whether this property is generic to reciprocal figures and to what extent reciprocal figures may be extended to integrable lattices of diverse combinatorics is currently under investigation.

\section{References}

[1] R.K. Bullough and P.J. Caudrey, eds, Solitons, Topics in Current Physics 17, Springer Verlag, Berlin (1980).

[2] S.E. Trullinger, V.E. Zakharov and V.L. Pokrovsky, eds, Solitons, Modern Problems in Condensed Matter Science 17, North-Holland, Amsterdam (1986).

[3] C. Hoenselaers and W. Dietz, eds, Solutions of Einstein's Equations: Techniques and Results, Lecture Notes in Physics, Springer Verlag, BerlinHeidelberg (1984).

[4] A. Hasegawa, Optical Solitons in Fibers, Springer Tract in Modern Physics 116, Springer Verlag, Berlin-New York (1989).

[5] H. Koch and H. Lübbig, eds, Single-Electron Tunneling and Mesoscopic Devices, Proceedings 4th International Conferences SQID, Springer Verlag, Berlin-New York (1991). 
[6] V.E. Zakharov, S.V. Manakov, S.P. Novikov and L.P. Pitaevskii, The Theory of Solitons: The Inverse Problem Method, Nauka, Moscow (1980); Plenum Press (1984).

[7] M.J. Ablowitz and P. Clarkson, Solitons, Nonlinear Evolution Equations and Inverse Scattering, Cambridge University Press (1991).

[8] C. Rogers and W.F. Shadwick, Bäcklund Transformations and Their Applications, Academic Press, New York (1982).

[9] V.B. Matveev and M.A. Salle, Darboux Transformations and Solitons, Springer Verlag, Berlin (1991).

[10] J.C. Maxwell, On reciprocal figures and diagrams of forces, Phil. Mag. Series 427 (1864) 250-261.

[11] W.D. Niven, ed, The Scientific Papers of James Clerk Maxwell, Dover, New York (1965).

[12] J.C. Maxwell, On reciprocal figures, frames and diagrams of forces, Trans. R. Soc. Edinburgh 26 (1870) 1-40.

[13] W.J.M. Rankine, Manual of Applied Mechanics, Griffin, London (1858).

[14] F. Jenkin, On the practical application of reciprocal figures to the calculation of strains on framework, Trans. R. Soc. Edinburgh 25 (1869) 441.

[15] F. Jenkin, On the application of graphic methods to the determination of the efficiency of machinery, Trans. R. Soc. Edinburgh 28 (1877) 1.

[16] T.S. Baynes, ed, The Encyclopaedia Britannica : A Dictionary of Arts, Sciences, and General Literature, 9th ed, Adam and Charles Black, Edinburgh (1875-1903).

[17] L. Cremona, Le Figure Reciproche Nella Statica Grafica, Milano (1872); English translation: Graphical Statics, Oxford University Press (1890).

[18] C. Culmann, Die Graphische Statik, Meyer und Zeller, Zürich (1866).

[19] A.K. Mackworth, Interpreting pictures of polyhedral scenes, Artificial Intelligence 4 (1973) 121-137.

[20] E.W. Elcock and D. Michie, eds, Machine Intelligence 8, Ellis Horwood Ltd., Chichester; Halsted Press, New York (1977).

[21] T. Miwa, On Hirota difference equations, Proc. Japan. Acad. A 58, 9-12 (1982).

[22] J.J.C. Nimmo and W.K. Schief, Superposition principles associated with the Moutard transformation: an integrable discretization of a 2+1dimensional sine-Gordon system, Proc. R. Soc. London A 453 (1997) 255279 . 
[23] J.J.C. Nimmo and W.K. Schief, An integrable discretization of a 2+1dimensional sine-Gordon equation, Stud. Appl. Math. 100 (1998) 295-309.

[24] W.K. Schief, Self-dual Einstein spaces via a permutability theorem for the Tzitzeica equation, Phys. Lett. A 223 (1996) 55-62.

[25] W.K. Schief, Self-dual Einstein spaces and a discrete Tzitzeica equation. A permutability theorem link, in Symmetries and Integrability of Difference Equations II, P. Clarkson and F. Nijhoff, eds, London Mathematical Society, Lecture Note Series 255, Cambridge University Press, Cambridge (1999) 137-148.

[26] A.I. Bobenko and W.K. Schief, Discrete indefinite affine spheres, in Discrete Integrable Geometry and Physics, A. Bobenko and R. Seiler, eds, Oxford University Press (1999) 113-138.

[27] A.I. Bobenko and W.K. Schief, Affine spheres: discretization via duality relations, J. Exp. Math. 8 (1999) 261-280.

[28] B.G. Konopelchenko and W.K. Schief, Menelaus' theorem Clifford configurations and inversive geometry of the Schwarzian KP hierarchy, submitted; arXiv:nlin.SI/0105023 (2001).

[29] R. Bow, Economics of Construction in Relation to Framed Structures, E. and F.N. Spon, London (1873).

[30] H. Crapo and W. Whiteley, Plane self stresses and projected polyhedra I: The basic pattern, Structural Topology 20 (1993) 55-78.

[31] D. Pedoe, A Course of Geometry, Cambridge University Press (1970).

[32] D.A. Brannan, M.F. Esplen and J.J. Gray, Geometry, Cambridge University Press (1999).

[33] F. Morley and F.V. Morley, Inversive Geometry, Chelsea Publ. Comp., New York (1954).

[34] N.W. Ashcroft and N.D. Mermin, Solid State Physics, Saunders College, Philadelphia (1976).

[35] M. Jimbo and T. Miwa, Solitons and infinite dimensional Lie algebras, Publ. RIMS, Kyoto Univ. 19, 943 (1983).

[36] B.G. Konopelchenko and C. Rogers, On a 2+1-dimensional nonlinear system of Loewner-type, Phys. Lett. A 152 (1991) 391-397.

[37] Th. Moutard, Sur la construction des équations de la forme $\frac{1}{z} \frac{\partial^{2} z}{\partial x \partial y}=\lambda(x, y)$ qui admettent une intégrale générale explicite, J. de l'Ecole Polytechnique, Cahier 45 (1878) 1-11. 
[38] J. Weiss, The Painlevé property for partial differential equations. II. Bäcklund transformations, Lax pairs and Schwarzian derivative, J. Math. Phys. 24, 1405-1413 (1983).

[39] L.V. Bogdanov and B.G. Konopelchenko, Analytic-bilinear approach to integrable hierarchies. I. Generalized KP hierarchy, J. Math. Phys. 39, 4683-4700 (1998).

[40] L.V. Bogdanov and B.G. Konopelchenko, Analytic-bilinear approach to integrable hierarchies. II. Multicomponent KP and 2D Toda lattice hierarchies, J. Math. Phys. 39, 4701-4728 (1998).

[41] A.I. Bobenko and R. Seiler, eds., Discrete Integrable Geometry and Physics, Clarendon Press, Oxford (1999).

[42] L.V. Bogdanov and B.G. Konopelchenko, Lattice and q-difference DarbouxZakharov-Manakov systems via $\bar{\partial}$-dressing method, J. Phys. A: Math. Gen. 28, L173-L178.

[43] A. Doliwa, Geometric discretisation of the Toda system, Phys. Lett. A 234, 187-192 (1997).

[44] G. Darboux, Leçons sur les systèmes orthogonaux et les coordonnées curvilignes, Gauthier-Villars, Paris (1910).

[45] B.G. Konopelchenko, W. Schief and C. Rogers, A 2+1-dimensional sineGordon system: its auto-Bäcklund transformation, Phys. Lett. A $\mathbf{1 7 2}$ (1992) 39-48.

[46] B.G. Konopelchenko and W.K. Schief, Three-dimensional integrable lattices in Euclidean spaces: conjugcy and orthogonality, Proc. R. Soc. London A 454 (1998) 3075-3104.

[47] G. Tzitzéica, Sur une nouvelle classe de surfaces, C. R. Acad. Sci. Paris 144 (1907) 1257-1259; Sur une classe de surfaces, ibid 146 (1908) 165-166.

[48] G. Tzitzéica, Sur une nouvelle classe de surfaces, C. R. Acad. Sci. Paris 150 (1910) 955-956; 1227-1229.

[49] W. Blaschke, Differentialgeometrie, Chelsea Publishing Company, New York, Reprinted (1967).

[50] A.V. Mikhailov, The reduction problem and the inverse scattering method, Physica D 3 (1981) 73-117.

[51] L.V. Ahlfors, Lectures on Quasiconformal Mappings, D. Van Nostrand Company, Princeton (1966).

[52] O. Lehto and K.I. Virtanen, Quasiconformal Mappings in the Plane, Springer Verlag, New York - Heidelberg (1973). 
[53] P. Caraman, n-Dimensional Quasiconformal (QCF) Mappings, Abacus Press, New Jersey (1974). 